PITT-97-333; LA-UR-98-??; LPTHE-98-05,PE-97-??

\title{
EVOLUTION OF INHOMOGENEOUS CONDENSATES: SELF-CONSISTENT VARIATIONAL APPROACH
}

\author{
D. Boyanovsky ${ }^{(a)}$, F. Cooper ${ }^{(b)}$, H. J. de Vega ${ }^{(c)}$, P. $\operatorname{Sodano}^{(d)}$ \\ (a) Department of Physics and Astronomy, University of Pittsburgh, Pittsburgh PA. 15260, U.S.A \\ (b) Theoretical Division, Los Alamos National Laboratory, Los Alamos, NM 87545, U.S.A. \\ (c) LPTHE, Université Pierre et Marie Curie (Paris VI) et Denis Diderot (Paris VII), Tour 16, \\ 1er. étage, 4, Place Jussieu, 75252 Paris, Cedex 05, France \\ (d)Dipartimento di Fisica and Sezione INFN, Università di Perugia, 06100 Perugia, Italia
}

(February 1998)

\begin{abstract}
We establish a self-consistent variational framework that allows us to study numerically the non-equilibrium evolution of non-perturbative inhomogeneous field configurations including quantum backreaction effects. After discussing the practical merits and disadvantages of different approaches we provide a closed set of local and renormalizable update equations that determine the dynamical evolution of inhomogeneous condensates and can be implemented numerically. These incorporate self-consistently the backreaction of quantum fluctuations and particle production. This program requires the solution of a self-consistent inhomogeneous problem to provide initial Cauchy data for the inhomogeneous condensates and Green's functions. We provide a simple solvable ansatz for such an initial value problem for the Sine Gordon and $\phi^{4}$ quantum field theories in one spatial dimension. We compare exact known results of the Sine Gordon model to this simple ansatz. We also study the linear sigma model in the large $N$ limit in three spatial dimensions as a microscopic model for pion production in ultrarelativistic collisions. We provide a solvable self-consistent ansatz for the initial value problem with cylindrical symmetry. For this case we also obtain a closed set of local and renormalized update equations that can be numerically implemented. A novel phenomenon of spinodal instabilities and pion production arises as a result of a Klein paradox for large amplitude inhomogeneous condensate configurations.
\end{abstract}

11.10.-z,11.10.Wx,11.10.Ex,25.75.-q

Typeset using REVTEX 


\section{INTRODUCTION}

The dynamical evolution of spatially inhomogeneous semiclassical field configurations along with quantum fluctuations that dress them play an important role in many relevant problems in high energy and condensed matter physics. Such situations are ubiquitous in the description of semiclassical processes such as baryogenesis via sphaleron decay [1, 2], bubble and droplet nucleation during supercooled phase transitions as it could be the case in QCD [3,4], the formation of topological defects [5,6] and the possibility of formation of disoriented chiral condensates [7-10]. Localized high energy and large amplitude field configurations are of particular relevance for modelling the dynamics of relativistic heavy ion collisions as was proposed for example by 111 in terms of collisions of Yang-Mills wave-packets. In this case an approximation to the dynamics of high initial multiplicity states would be to provide an initial value problem with a particular initial condition on the field at some proper time surface. By proposing a large amplitude field configuration localized in space in the form of a semiclassical coherent state, the features associated with an initial state of large energy density and high multiplicity are captured. For example such field could be a strong color field or electric field whose relaxation will result in high multiplicity particle production [11].

The dynamics of coherent structures and semiclassical field configurations is also of theoretical and experimental relevance in condensed matter physics within the context of transport phenomena mediated by soliton excitations in quasi-one dimensional systems [12 [14].

Although there have been several studies of real-time dynamics of classical field configurations within the context of sphaleron decay [2], Non-Abelian wave-packet collisions [11] and coherent structures in scalar field theories [15] including classical [16 18] and semiclassical [19] studies of disoriented chiral condensates, there have been very few and limited attempts to incorporate consistently the effects of quantum fluctuations upon the dynamics of semiclassical inhomogeneous configurations and mainly in one spatial dimension [20]. Whereas the dynamics of inhomogeneous field configurations had been studied in the small amplitude regime [22], the important regime of large amplitude and non-perturbative field configurations is still not understood.

Recently there has been tremendous progress in studying the non-equilibrium dynamics of high energy and high multiplicity quantum states including self-consistently quantum fluctuations in the case of spatial or spatial rapidity homogeneity to study particle production in strong fields, dynamics of phase transitions and relaxation and the dynamics of inflation [21]- 35]. In particular these methods had been used to study the formation of disoriented chiral condensates, and pion production and relaxation during the chiral phase transition in low-energy phenomenological models [33,34] and more recently initial quantum states of large multiplicity distributions with localized momenta [35].

These studies rely on an implementation of variational and large $N$ [23]- 25] approximations generalized to strongly out of equilibrium situations. These approximations [36]39] are specially suited for studying non-perturbative phenomena and in particular the large $N$ approximation is amenable to consistent improvement [24,25].

Since these methods allow one to study the full quantum evolution in a non-perturbative manner they are specially tailored to studying the non-equilibrium evolution of initial inhomogeneous quantum states of large energy including quantum back-reaction effects.

This is specially important in the modelling of relativistic heavy ion collisions in a man- 
ner that allows one to include the effects of quantum backreaction and non-perturbative particle production. A useful description of the dynamical evolution in relativistic heavy ion collisions is based on a hydrodynamic picture in terms of a fluid that is described in local thermodynamic equilibrium by a local energy momentum tensor and an equation of state 40,41 . Although such a description is not only physically compelling but also experimentally consistent at the present energies [3,42], it is at best phenomenological and not justified from a microscopic theory. The hydrodynamic description relies on the assumption that as a result of the collision a large amount of energy is deposited in a small region of space-time. Boost invariant hydrodynamics [40,41] should emerge when the energy density is much larger than the typical hadronic masses, and is a result of hydrodynamic scaling when the resulting pancake width goes to zero with center of mass energy. In this scaling limit the relevant degrees of freedom for describing the expansion are the fluid proper time and fluid rapidity.

Although trying to study the emergence of boost invariant hydrodynamics within the framework of QCD is at this stage an impossible task, the features of hydrodynamical evolution and particle production are rather robust and should be captured by a microscopic albeit semi-phenomenological theory.

In this article we begin our study of the non-equilibrium dynamics of inhomogeneous field configurations of large amplitude including the back-reaction of quantum fluctuations self-consistently and non-perturbatively. The focus and goal of this program is to study the relaxation of a strongly out of equilibrium inhomogeneous initial state, the description of particle production and the emergence of a hydrodynamical description from an ab initio full quantum evolution in a microscopic, phenomenologically inspired quantum field theory.

This article is devoted to setting up the variational self-consistent problem and to studying the subtle but important issues of renormalization. The implementation of variational or large $N$ approximations to study the dynamics requires: i) to determine the Cauchy data for the inhomogeneous expectation value of the field as well as the relevant Green's functions that account for backreaction. Since both the variational and the large $N$ approximations lead to a self-consistent condition, the Cauchy data must be determined self-consistently. The solution to this self-consistent initial value problem for inhomogeneous field configurations is typically an extremely difficult and numerically intensive task. ii) setting up a closed set of local renormalized update equations that can be numerically implemented in current computers. Both the locality as well as renormalizability aspects are extremely important: current computational capabilities cannot handle non-local update equations because they are memory intensive. The numerical evolution will be implemented on a space-time mesh and the results should be insensitive to the mesh size, this requires renormalized evolution equations, which is also important to improve numerical stability. This issue is clearly very important for the quantum backreaction because the Green's functions that are necessary have short distance singularities that must be renormalized.

iii) Finally the numerical implementation of the update equations obtained in ii) starting from the initial data provided by the first step.

In this work we seek to provide the first two steps to implement this program, leaving the numerical implementation to a forthcoming article.

The coupled evolution equations that we find more suitable for a numerical approach in the self-consistent Hartree and large $\mathrm{N}$ approximations are summarized below in eqns. 
(6.1-6.5).

We focus on studying the initial value problem and the update equations first in relevant cases in $1+1$ dimensions not only because it is a simpler setting but also because it is a relevant problem not only in quantum field theory but also in condensed matter physics in the case of quasi-one dimensional systems. By treating these examples in one spatial dimension we determine a strategy to set up the self-consistent initial value problem that can be implemented for particular three dimensional geometries relevant to the description of 'colliding pancakes' in heavy ion collisions. This strategy is based on mapping the initial value problem (in terms of the variational parameters) onto a Schrödinger-like problem whose spectrum of eigenvectors and eigenvalues is exactly known.

We then move on to the linear sigma model description of pion physics and implement a large $N$ self-consistent approximation and the strategy developed in the one dimensional case to set up the Cauchy data. In this case we find some novel phenomena associated with spinodal instabilities for large amplitude field configurations. Finally we address the issue of renormalization and obtain the (semi) local update equations.

The article is organized as follows: in section II we introduce the different formulations to implement the variational and Hartree approximations, discussing the advantages and disadvantages of each of the methods and determine the method that we find the most suitable for numerical implementation at this stage.

Section III is devoted to a description of the initial value problem. Section IV presents a detailed analysis of the self-consistent initial value problem for the cases of the Sine-Gordon and $\phi^{4}$ theories in $1+1$ space-time dimensions. After analyzing the vacuum structure in the variational approximation we propose simple self-consistent Ansatzse for the Cauchy data for both topological (kink) and pulse-like (lumps) field configurations by establishing a correspondence between the initial value problem in terms of the variational parameters and the eigenvalue problem of a Schrödinger operator whose spectrum is exactly known. The renormalization aspects are studied in detail. These examples are relevant for studying the dynamics of solitons dressed by the quantum fluctuations in $1+1$ dimensions and to study the relaxation of large amplitude field configurations via meson production.

In section $\mathrm{V}$ we study the linear sigma model description of low energy pion phenomenology by implementing a large $N$ approximation in $3+1$ dimensions. We find that the leading order in the large $\mathrm{N}$ is similar to a Hartree factorization which allows us to use the formulation presented in section II.

We use the results of the $1+1$ dimensional case to set up self-consistent Cauchy data for an initial inhomogeneous configuration with cylindrical symmetry that could model the initial state after the collision of Lorentz contracted 'pancakes'. The subtle renormalization aspects are discussed in detail and we find a phenomenon akin to the Klein paradox that results in spinodal instabilities for large amplitude inhomogeneous configurations.

In section VI the dynamical evolution equations are summarized in terms of fully renormalized quantities and the initial data on all of the Green's functions that are needed for the update is determined. Section VII proposes a numerical strategy for an implementation of the dynamics. We summarize our strategy for the present and future work in the conclusions. 


\section{NON-EQUILIBRIUM DYNAMICS: METHODS}

There are several alternative ways to establish a variational self-consistent formulation of the dynamical evolution, and although all of them are equivalent, some particular implementation may present some practical advantages over others.

Thus we begin the program of setting up the variational self-consistent formulation by first studying the different implementations, discussing their merits and drawbacks, and choosing a particular formulation that would lead to an economical numerical implementation.

\section{A. Time Dependent Variational Principle}

The method for obtaining a time dependent variational approximation to the nonequilibrium dynamics in quantum field theory is to start with Dirac's variational principle for obtaining the functional Schrödinger equation.

This variational approach is implemented by proposing Gaussian wave functionals [24, 36]- 39], whose parameters are determined self-consistently. This approach leads to the time dependent Hartree approximation, and becomes exact in the case of a scalar field in the vector representation of the $O(N)$ group in the large $N$ limit 24, 25].

Dirac's variational principle proposes to extremize the effective action

$$
\Gamma=\int d t<\Psi\left|i \frac{\partial}{\partial t}-H\right| \Psi>;\langle\Psi \mid \Psi\rangle=1
$$

and leads directly to the Schrödinger equation:

$$
\delta \Gamma=0 \rightarrow\left\{i \frac{\partial}{\partial t}-H\right\} \mid \Psi>=0
$$

In the Schrödinger $(\varphi)$ representation, the wave-functionals are given by

$$
\Psi[\varphi, t]=<\varphi \mid \Psi>
$$

and the functional Schrödinger equation becomes

$$
i \frac{\partial}{\partial t} \Psi[\varphi, t]=\int d^{d} x\left\{-\frac{1}{2} \frac{\delta^{2}}{\delta \varphi(x)^{2}}+\frac{1}{2} \nabla \varphi(x) \nabla \varphi(x)+V[\varphi]\right\} \Psi[\varphi, t]
$$

Where we have specified $d$-spatial dimensions. In later sections we will address $d=1$ and $d=3$ separately in specific examples.

In this representation the variational approach is implemented by proposing a Gaussian or Hartree trial wave functional [24,36]- [39]:

$$
\begin{aligned}
\Psi_{v}[\varphi, t]= & N(t) \exp \left\{-\int d^{d} x \int d^{d} y\left\{\left(\varphi(\vec{x})-\phi_{c}(\vec{x}, t)\right) \mathcal{K}(\vec{x}, \vec{y} ; t)\left(\varphi(\vec{y})-\phi_{c}(\vec{y}, t)\right)\right\}+\right. \\
& \left.i \int d^{d} x \pi_{c}(x, t)\left(\varphi(x)-\phi_{c}(x, t)\right)\right\} \\
\mathcal{K}(\vec{x}, \vec{y} ; t)= & G^{-1}(x, y, t) / 4-i \Sigma(x, y, t)
\end{aligned}
$$


where in 2.5$)$ we have written the kernel $\mathcal{K}(\vec{x}, \vec{y} ; t)$ explicitly in terms of its real $G$ and imaginary $\Sigma$ parts. The real quantities $\varphi_{c} ; G ; \Sigma ; \pi_{c}$ are variational and have the following meaning:

$$
\begin{aligned}
& \phi_{c}(x, t)=<\Psi_{v}|\varphi(x)| \Psi_{v}>; \pi_{c}(x, t)=<\Psi_{v}|-i \delta / \delta \varphi(x)| \Psi_{v}> \\
& G(x, y, t)=<\Psi_{v}|\varphi(x) \varphi(y)| \Psi_{v}>-\phi_{c}(x, t) \phi_{c}(y, t) \\
& <\Psi_{v}\left|\varphi(x, t) \frac{1}{i} \frac{\delta}{\delta \varphi(y, t)}\right| \Psi_{v}>-\phi_{c}(x, t) \pi_{c}(y, t)=2 \int d^{d} z G(x, z ; t) \Sigma(z, y ; t)+\frac{i}{2} \delta^{3}(x-y)
\end{aligned}
$$

The subindex $v$ stands for variational.

In terms of these parameters the effective action for the trial wave functional becomes

$$
\begin{aligned}
\Gamma\left(\phi_{c}, \pi_{c}, G, \Sigma\right) & =\int d t<\Psi_{v}|i \partial / \partial t-H| \Psi_{v}> \\
& =\int d t d^{d} x\left\{\pi_{c}(x, t) \frac{\partial \phi_{c}(x, t)}{\partial t}+\int d t d^{d} x d^{d} y \Sigma(x, y ; t) \frac{\partial G(x, y ; t)}{\partial t}\right\} \\
& -\int d t<H>
\end{aligned}
$$

where

$$
\begin{aligned}
<H> & =\int d^{d} x\left\{\frac{1}{2} \pi^{2}+2[\Sigma G \Sigma](x, x)+\frac{1}{8} G^{-1}(x, x)+\frac{1}{2}(\nabla \varphi)^{2}\right. \\
& \left.+\frac{1}{2} \lim _{x \rightarrow y} \nabla_{x} \nabla_{y} G(x, y)+<V[\varphi(x)]>\right\} .
\end{aligned}
$$

$<H>$ is a constant and is a first integral of the motion. The equations that follow by varying the effective action with respect to the variational parameters are:

$$
\begin{aligned}
\dot{\pi}_{c}(x, t) & =\nabla^{2} \phi_{c}(x, t)-\frac{\partial<V>}{\partial \phi_{c}}(\vec{x}, t) \\
\dot{\phi}_{c}(x, t) & =\pi_{c}(x, t) \\
\dot{G}(x, y ; t) & =2 \int d^{d} z[\Sigma(x, z) G(z, y)+G(x, z) \Sigma(z, y)] \\
\dot{\Sigma}(x, y ; t) & =\int d^{d} z\left[-2 \Sigma(x, z) \Sigma(z, y)+\frac{1}{8} G^{-1}(x, z) G^{-1}(z, y)\right] \\
& +\left[\frac{1}{2} \nabla_{x}^{2}-\frac{\partial<V>}{\partial G}(\vec{x}, t)\right] \delta(x-y)
\end{aligned}
$$

These equations form a closed system and are the Time Dependent Hartree (Fock) equations (TDHF). They have the drawback that they are non-local and involve not only the Green's function $G(x, y ; t)$ but also its inverse, furthermore the renormalizability aspects of the dynamical equations are not transparent in this formulation. A numerical implementation of an initial value problem must provide Cauchy data for the variational parameters and implement a numerical update procedure that yields finite quantities, i.e. is free of short distance divergences. 
There is an equilibrium by product of this method in the form of the variational effective potential for the case of static homogeneous configurations which is given by [36,39]

$$
V_{e f f}\left(\phi_{c}\right)=\frac{1}{\Omega}<\Psi|H| \Psi>;<\Psi|\varphi| \Psi>=\phi_{c} ;<\Psi \mid \Psi>=1
$$

with $\Omega$ being the quantization volume and $\langle H\rangle$ is given in equation (2.8). This effective potential is useful to establish the phase structure of the particular theory in this Gaussian variational approximation.

\section{B. Method of Equal- time Green's Functions}

The update equations for the kernels that appear in the Time Dependent Hartree variational wave function are non-local as displayed by the set of equations (2.9). We can trade this non-locality by increasing the number of equations. This is achieved by considering the first order update equations for the equal time Green's functions in terms of the Heisenberg field operator $\varphi$ and its canonically conjugate momentum $\pi=\dot{\varphi}$. Consider the evolution equation of the following quantities,

$$
\begin{aligned}
& \phi_{c}(\vec{x}, t)=<\varphi(\vec{x}, t)>; \pi_{c}(\vec{x}, t)=<\dot{\varphi}(\vec{x}, t)> \\
& G(\vec{x}, \vec{y}, t)=<\varphi(\vec{x}, t) \varphi(\vec{y}, t)>-\phi_{c}(\vec{x}, t) \phi_{c}(\vec{y}, t) \\
& D(\vec{x}, \vec{y}, t)=<\varphi(\vec{x}, t) \pi(\vec{y}, t)>-\phi_{c}(\vec{x}, t) \pi_{c}(\vec{y}, t) \\
& \tilde{D}(\vec{x}, \vec{y}, t)=<\pi(\vec{x}, t) \varphi(\vec{y}, t)>-\phi_{c}(\vec{y}, t) \pi_{c}(\vec{x}, t)=D(\vec{y}, \vec{x}, t)-i \delta^{d}(\vec{x}-\vec{y}) \\
& K(\vec{x}, \vec{y}, t)=<\dot{\varphi}(\vec{x}, t) \dot{\varphi}(\vec{y}, t)>-\pi_{c}(\vec{x}, t) \pi_{c}(\vec{y}, t) .
\end{aligned}
$$

We can relate $D \tilde{D}$ and $K$ to $G$ and $\Sigma$ as follows:

$$
\begin{gathered}
D(x, y ; t)+\tilde{D}(y, x ; t)=4 \int d^{d} z G(x, z ; t) \Sigma(z, y ; t) \\
\tilde{D}(x, y ; t)+D(y, x, t)=4 \int d^{d} z \Sigma(x, z ; t) G(z, y ; t) \\
K(x, y ; t)=\frac{1}{4} G^{-1}(x, y ; t)+4 \int d^{d} z_{1} d^{d} z_{2} \Sigma\left(x, z_{1} ; t\right) G\left(z_{1}, z_{2} ; t\right) \Sigma\left(z_{2}, y ; t\right)
\end{gathered}
$$

This last equation, eq. (2.13) shows that $K$ is not an independent quantity.

All of the expectation values are with respect to the initial Gaussian trial wave-functional or density matrix.

The time derivatives of operators are obtained from the Heisenberg equations with the Hamiltonian

$$
H=\int d^{d} x\left\{\frac{1}{2} \pi(x)^{2}+\frac{1}{2} \nabla \varphi \cdot \nabla \varphi+\frac{\mu^{2}}{2} \varphi^{2}+V[\varphi(x)]\right\}
$$

and the canonical commutation relations between the field $\varphi$ and its canonical conjugate momentum $\pi$. 
These lead to the Heisenberg operator equations of motion:

$$
\begin{aligned}
& \pi(x, t)=\dot{\varphi}(x, t) \\
& \dot{\pi}(x, t)=\nabla^{2} \varphi(x, t)-\partial V / \partial \varphi(x, t)
\end{aligned}
$$

The evolution equation for $\phi_{c}$ is as given before

$$
\partial^{2} \phi_{c}(x, t)+<\frac{\partial V}{\partial \varphi(x, t)}>=0
$$

and we obtain the following coupled equations for the kernels $G, D, \tilde{D}$ and $K$ :

$$
\begin{gathered}
\dot{G}(\vec{x}, \vec{y} ; t)=D(\vec{x}, \vec{y} ; t)+\tilde{D}(\vec{x}, \vec{y} ; t) \\
\dot{D}(\vec{x}, \vec{y} ; t)=K(\vec{x}, \vec{y} ; t)+\nabla_{y}^{2} G(\vec{x}, \vec{y} ; t)-<\left\{\varphi(\vec{x}, t)-\phi_{c}(\vec{x}, t)\right\} \frac{\partial V}{\partial \varphi(\vec{y}, t)}> \\
\dot{K}(\vec{x}, \vec{y} ; t)=\nabla_{x}^{2} D(\vec{x}, \vec{y} ; t)+\nabla_{y}^{2} \tilde{D}(\vec{x}, \vec{y} ; t) \\
-<\left\{\dot{\varphi}(x, t)-\dot{\phi}_{c}(x, t)\right\} \frac{\partial V}{\partial \varphi(y, t)}>+<\frac{\partial V}{\partial \varphi(x, t)}\left\{\dot{\varphi}(y, t)-\dot{\phi}_{c}(y, t)\right\}> \\
+<\frac{\partial V}{\partial \varphi(y, t)}\left\{\dot{\varphi}(x, t)-\dot{\phi}_{c}(x, t)\right\}>+<\left\{\dot{\varphi}(y, t)-\dot{\phi}_{c}(y, t)\right\} \frac{\partial V}{\partial \varphi(x, t)}>
\end{gathered}
$$

The set of eqns. (2.16 2.19$)$ is equivalent to the TDHF equations (2.9).

If we evaluate all the equations of motion values using a Gaussian Density Matrix, the equations for $\phi_{c}, G, D$ and $K$ close, which is the usual truncation of the Dyson equations in a mean field approximation.

We would like to show that the equation for $K$ guarantees in the update that the quantity

$$
N(x, y, t) \equiv K(x, y ; t)-\frac{1}{4} G^{-1}(x, y ; t)-4 \int d^{d} z_{1} d^{d} z_{2} \Sigma\left(x, z_{1} ; t\right) G\left(z_{1}, z_{2} ; t\right) \Sigma\left(z_{2}, y ; t\right)
$$

is conserved in time. In the spatially homogeneous case we showed [30] that $N(x, y, t)$ was related to the initial number of particles present at $t=0$. If we are in a pure state such as one described by a Gaussian wave function, $\left\langle a^{\dagger} a\right\rangle=0$ at $t=0$ as will be discussed below. A nonzero value of $N$ can be obtained if we start with a Gaussian density matrix as discussed in [27].

First one can show explicitly, for the field theories discussed in this article, using the equations of motion for $G$ and $\Sigma$ that for $K$ defined by eq.(2.13) $K$ obeys eq. (2.19) so that the equation for $K$ is automatically satisfied if we solve the TDHF equations (2.9). Conversely, we can rewrite the equation for $N(2.20)$ in terms of $D$ and $\tilde{D}$ using the relation:

$\Sigma(x, y ; t)=\frac{1}{4} \int d z G^{-1}(x, z ; t)\{D(z, y ; t)+\tilde{D}(y, z ; t)\} \equiv \frac{1}{4} \int d^{d} z\{\tilde{D}(x, z ; t)+D(z, x ; t)\} G^{-1}(z, y ; t)$. 
Using the equations of motion for $G D \tilde{D}$ and $K$ one can then show that $\frac{d N}{d t}=0$. Thus if we choose our initial condition on $\mathrm{K}$ to satisfy the constraint:

$$
\begin{aligned}
& K(x, y ; t=0)=\frac{1}{4} G^{-1}(x, y ; t=0) \\
& +\frac{1}{4} \int d^{d} z_{1} d^{d} z_{2}\left\{\tilde{D}\left(x, z_{1} ; 0\right)+D\left(z_{1}, x ; 0\right)\right\} G^{-1}\left(z_{1}, z_{2} ; 0\right)\left\{D\left(z_{2}, y ; 0\right)+\tilde{D}\left(y, z_{2} ; 0\right)\right\}
\end{aligned}
$$

then the conservation law (which is equivalent to eq. (2.19) guarantees that this constraint is observed at all times.

There is a definite advantage in these update equations over the TDHF ones, as we will see in specific examples below. The equal time Green's function equations lead to a spatially local update (apart from gradients), which is quite an advance over the previous (TDHF) case which required non local updates as well as matrix inversions and therefore is computationally more intensive. The small price one pays is to increase the number of variables by one.

\section{Covariant approach to mean field theory}

The covariant approach to mean field theory, arises from a self consistent truncation of the Schwinger Dyson hierarchy of equations for the N-point Green's functions to just the first two equations: namely an equation for the one and two particle connected Green's Functions. This can be accomplished directly in terms of the Dyson equations (as we do here), or by introducing a quadratic constraint field in a path integral approach and performing a loop expansion with respect to the constraint field [23].

Starting from the Lagrangian

$$
L=\int d^{d} x d t\left\{\frac{1}{2} \partial_{\mu} \varphi \partial^{\mu} \varphi-V[\varphi]\right\}
$$

we want to obtain the equations of motion for the expectation values

$$
\phi_{c}(\vec{x}, t)=\operatorname{Tr} \rho \varphi(\vec{x}, t)=<\varphi(\vec{x}, t)>.
$$

and the two time Wightman functions : $G^{<,>}\left(\vec{x}, \vec{y} ; t, t^{\prime}\right)$ given by

$$
\begin{aligned}
& G^{>}\left(\vec{x}, \vec{y} ; t, t^{\prime}\right)=\operatorname{Tr}\left[\rho \varphi(\vec{x}, t) \varphi\left(\vec{y}, t^{\prime}\right)\right]-\phi_{c}(\vec{x}, t) \phi_{c}\left(\vec{y}, t^{\prime}\right) \\
& G^{<}\left(\vec{x}, \vec{y} ; t, t^{\prime}\right)=\operatorname{Tr}\left[\rho \varphi(\vec{y}, t) \varphi\left(\vec{x}, t^{\prime}\right)\right]-\phi_{c}(\vec{y}, t) \phi_{c}\left(\vec{x}, t^{\prime}\right)
\end{aligned}
$$

Expectation values are in the initial density matrix $\rho$. The various Green's Functions can be constructed from the two Wightman functions. In the mean field approximation, the exact equation of motion for $\phi_{c}$ is:

$$
\partial^{2} \phi_{c}(x, t)+<\frac{\partial V[\varphi]}{\partial \varphi(x, t)}>=0
$$


which is approximated by replacing the exact expectation value by the expectation value using the Gaussian trial wave-functional. Making the same approximation to the exact equation for the two point function one obtains for the Green's function:

$$
\left\{\partial^{2}+<\frac{\partial^{2} V[\varphi]}{(\partial \varphi(x, t))^{2}}>\right\} G\left(\vec{x}, \vec{y} ; t, t^{\prime}\right)=\delta^{d}(\vec{x}-\vec{y}) \delta_{\mathcal{C}}\left(t-t^{\prime}\right)
$$

Here because the boundary conditions pertain to an initial value problem, the delta function is defined on the Closed Time Path (CTP) contour of the Schwinger-Keldysh formulation [44. Namely

$$
\delta_{\mathcal{C}}\left(t-t^{\prime}\right)=\frac{d \Theta_{\mathcal{C}}\left(t, t^{\prime}\right)}{d t}
$$

where

The expectation value is obtained using the Gaussian density matrix. Since the expectation values obtained in the Gaussian trial state depend only on $\phi_{c}$ and $G$ the system of coupled Green's function equations truncates at this level. The causal Green's function is related to the Wightman functions defined above as follows:

$$
G\left(x, y, t, t^{\prime}\right)=\Theta_{\mathcal{C}}\left(t, t^{\prime}\right) G^{>}\left(x, y, t, t^{\prime}\right)+\Theta_{\mathcal{C}}\left(t^{\prime}, t\right) G^{<}\left(x, y, t, t^{\prime}\right) .
$$

In the covariant approach one needs two time information about the Green's function. Thus if we directly solve this equation, starting from the initial data at $t=t^{\prime}=0$, it would be computationally much more storage intensive than considering the set of coupled equal time Green's functions. One way to solve this Green's function equation and simultaneously handle the mass renormalization problem is to convert the partial differential equation into an integral equation. Defining $M^{2}(x, t)$ via

$$
M^{2}(x, t) \equiv<\frac{\partial^{2} V[\varphi]}{(\partial \varphi(x, t))^{2}}>
$$

and calling $m_{r}^{2}$ the mass in the Hartree approximation in the vacuum sector, then we have that

$$
G\left(x, y, t, t^{\prime}\right)=G_{0}\left(x, y, t, t^{\prime}\right)-\int d^{d} z \int_{\mathcal{C}} d t^{\prime \prime} G_{0}\left(x, z, t, t^{\prime \prime}\right) \Delta M^{2}\left(z, t^{\prime \prime}\right) G\left(z, y, t^{\prime \prime}, t^{\prime}\right)
$$

where

$$
\left.\Delta M^{2}(x, t)\right)=M^{2}(x, t)-m_{r}^{2}
$$

and

$$
\int_{\mathcal{C}} d t=\int_{0, \mathcal{C}_{+}}^{\infty} d t-\int_{0, \mathcal{C}_{-}}^{\infty} d t
$$

The CTP formalism [44] guarantees causality so that $t^{\prime \prime}<\left\{t, t^{\prime}\right\}$. Solving this integral equation numerically is quite storage intensive. Here $G_{0}$ is the free field theory CTP Green's function for a particle with mass $m_{r}^{2}$. Integral equations of this type and their numerical solution are discussed in [43]. 
To circumvent these storage problems, one can use another strategy for updating $G$ which relies on finding a complete set of solutions for the quantum fluctuation field out of which the Green's function can be constructed. That is if we have a complete orthonormal set of solutions $\psi_{k}(x, t)$ to the equation:

$$
\left\{\partial^{2}+M^{2}(x, t)\right\} \psi_{k}(x, t)=0,
$$

where the scalar product is defined by

$$
<f, g>=i \int d^{d} x\left[f^{*}(x, t) \frac{\partial g(x, t)}{\partial t}-g(x, t) \frac{\partial f^{*}(x, t)}{\partial t}\right]
$$

then we can expand the fluctuation field $\hat{\psi}$ (where $\varphi=\phi_{c}+\hat{\psi}$ ) as follows:

$$
\hat{\psi}(x, t)=\sum_{k}\left\{a_{k} \psi_{k}(x, t)+a_{k}^{\dagger} \psi_{k}^{*}(x, t)\right\}
$$

with the canonical momentum $\pi(x, t)$ given by:

$$
\pi(x, t)=\sum_{k}\left\{a_{k} \partial_{t} \psi_{k}(x, t)+a_{k}^{\dagger} \partial_{t} \psi_{k}^{*}(x, t)\right\}
$$

(for continuum modes $\sum_{k} \equiv \int \frac{d^{d} k}{(2 \pi)^{d}}$ ).

The mode functions satisfy the orthogonality conditions:

$$
<\psi_{k}, \psi_{l}>=\delta(k-l) ; \quad<\psi_{k}^{*}, \psi_{l}>=0
$$

Assuming the usual commutation relations for the creation and annihilation operators:

$$
\left[a_{k}, a_{l}^{\dagger}\right]=\delta_{k l} ; \quad\left[a_{k}, a_{l}\right]=\left[a_{k}^{\dagger}, a_{l}^{\dagger}\right]=0 .
$$

(where $\delta_{k l} \equiv(2 \pi)^{d} \delta^{d}(k-l)$ for continuum modes) leads to the closure or completeness condition:

$$
i \sum_{k}\left[\psi_{k}^{*}(x, t) \frac{\partial \psi_{k}(y, t)}{\partial t}-\psi_{k}(x, t) \frac{\partial \psi_{k}^{*}(y, t)}{\partial t}\right]=\delta(x-y) .
$$

One can verify using the equation of motion (2.31) for the modes, that if the modes are orthogonal at $t=0$ that they stay orthogonal at all times.

For the case of spatial homogeneity, it is quite simple to find a complete set of orthonormal solutions to the classical field problem at all times [26]- [35]. The mode equations become for spatially homogeneous problems

$$
\left\{\partial^{2}+M^{2}(t)\right\} \psi_{k}(x, t)=0
$$

Letting

$$
\psi_{k}(x, t)=f_{k}(t) e^{i k x}
$$

we find the $f_{k}$ obey the ordinary differential equation: 


$$
\ddot{f}_{k}+\left\{k^{2}+M^{2}(t)\right\} f_{k}=0
$$

In terms of the $f_{k}$ one then has that the fluctuation field can be constructed at all times as:

$$
\hat{\psi}(x, t)=\sum_{k}\left\{a_{k} f_{k}(t) e^{i k x}+a_{k}^{\dagger} f_{k}^{*}(t) e^{-i k x}\right\}
$$

The closure relationship is then satisfied as long as $f_{k}$ obey the Wronskian condition at $t=0$ :

$$
f_{k} \frac{d f_{k}^{*}}{d t}-f_{k}^{*} \frac{d f_{k}}{d t}=i
$$

Renormalization constraints require that for large values of $k$ the modes $f_{k}$ differ in a prescribed fashion from the free field ones. The renormalization can be performed by studying the WKB expansion of the mode functions and isolating the divergences as discussed in several references: [45,29]. The initial values of $f_{k}$ and $\dot{f}_{k}$ are usually chosen so that the initial state is either in an adiabatic vacuum state or a thermal state with respect to the adiabatic vacuum as discussed in [45,29].

All the non-equilibrium Green's functions, can be constructed in terms of the Wightman function

$$
G^{>}\left(\vec{x}, \vec{y}, t, t^{\prime}\right)=<\hat{\psi}(\vec{x}, t) \hat{\psi}\left(\vec{y}, t^{\prime}\right)>
$$

in the usual fashion.

In the spatially inhomogeneous case, the mode functions obey the partial differential equation:

$$
\left\{\partial^{2}+M^{2}(x, t)\right\} \psi_{k}(x, t)=0
$$

$M^{2}(x, t)$ is determined from the same modes that solve this equation, thus it is necessary, in order to get started at $t=0$ to find a self consistent choice for $M^{2}(x, t=0)$ and the mode functions. This will allow us to determine the initial conditions for the partial differential equations for each mode. This aspect of the problem will be addressed in the following sections. The second problem in this approach is to find efficient symplectic integration methods which will allow us to simultaneously update many partial differential equations, each of which is similar to updating the time dependent Schrödinger equation. The way that the initial conditions are handled is by assuming at $t=0$ that we can write:

$$
\psi_{k}(x, t)=\frac{e^{-i \omega_{k} t}}{\left(2 \omega_{k}\right)^{1 / 2}} g_{k}(x) ; g_{k}^{*}=g_{-k}
$$

Then the closure relationship becomes the completeness relation:

$$
\sum_{k} g_{k}^{*}(x) g_{k}(y)=\delta(x-y)
$$

and the $g_{k}$ obey a Schrödinger equation.

$$
\left\{-\nabla^{2}+M^{2}(x, t=0)\right\} g_{k}(x)=\omega_{k}^{2} g_{k}(x) .
$$


The covariant approach to mean field theory is often the first term in a systematic expansion of the full field theory, so that the advantage here is that one can determine the next order corrections and see how long the mean field approximation is valid. This has been carried out in a simple quantum mechanical example in 43]. The standard method for doing the systematic expansion is to start with the path integral and use closed time path boundary conditions to insure causality. This is discussed in great detail in [24,25]. The advantage of having a mode function decomposition for the Green's functions is that subtractions necessary for renormalization can be made on a mode by mode basis, which dramatically improves the convergence of the mode sums.

\section{Operator and Closed Time Path Methods:}

A convenient implementation of the Gaussian variational approximation at the operator level begins with the Lagrangian formulation, shifting the field by its expectation value and implementing a Hartree-like factorization of non-linear interaction terms in order to make the Lagrangian linear plus quadratic. The linear terms in the fluctuation are required to vanish, leading to an equation of motion for the expectation value, the remaining Lagrangian is a quadratic form in terms of the fluctuations. Passing to a Hamiltonian formulation for the operator fluctuations the Heisenberg equations of motion for the operators is obtained, and the expectation values are now understood in the Heisenberg representation in the initial state.

Specifically, for a Lagrangian density of the form

$$
\mathcal{L}[\varphi]=\frac{1}{2} \partial_{\mu} \varphi \partial^{\mu} \varphi-V(\varphi)
$$

(where we have omitted the linear coupling to sources), the Gaussian approximation is implemented in the following steps: i) shift the field $\varphi$ and its expectation value

$$
\begin{aligned}
\hat{\varphi}(\vec{x}, t) & =\varphi_{c}(\vec{x}, t)+\hat{\psi}(\vec{x}, t) \\
\langle\hat{\psi}(\vec{x}, t)\rangle & =0
\end{aligned}
$$

ii) After this shift the potential has in general even and odd powers of the fluctuation $\psi$,

$$
\begin{aligned}
V\left[\varphi_{c}+\psi\right] & =V^{e}\left[\varphi_{c}, \psi\right]+V^{o}\left[\varphi_{c}, \psi\right] \\
V^{e}\left[\varphi_{c}, \psi\right] & =V\left[\varphi_{c}\right]+\psi^{2}\left(\frac{\partial^{2} V(\varphi)}{\partial \varphi^{2}}\right)_{\varphi=\varphi_{c}+\psi} \\
V^{o}\left[\varphi_{c}, \psi\right] & =\psi\left(\frac{\partial V(\varphi)}{\partial \varphi}\right)_{\varphi=\varphi_{c}+\psi}
\end{aligned}
$$

The Hartree factorization replaces the even and odd parts by

$$
\begin{aligned}
& V_{H}^{e}\left[\varphi_{c}, \psi\right] \rightarrow V\left[\varphi_{c}\right]+\psi^{2} M^{2}(\vec{x}, t)+\mathcal{C}(\vec{x}, t) \\
& M^{2}(x, t)=\left\langle\left.\frac{\partial^{2} V(\varphi)}{\partial \varphi^{2}}\right|_{\varphi=\varphi_{c}+\psi}\right\rangle \\
& V_{H}^{o}\left[\varphi_{c}, \psi\right]=\psi \mathcal{V}(\vec{x}, t) \\
& \mathcal{V}(\vec{x}, t)=\left\langle\left.\frac{\partial V(\varphi)}{\partial \varphi}\right|_{\Phi=\varphi_{c}+\psi}\right\rangle
\end{aligned}
$$


Where the expectation values will be obtained self-consistently. The field independent term $\mathcal{C}$ is obtained by requesting that the expectation value of the potential equals the expectation value of its Hartree factorized form.

Upon integration by parts in the resulting action, the linear term in the fluctuation $\psi$ is requested to vanish to ensure the condition (2.45), leading to the equation of motion for the expectation value

$$
\frac{\partial^{2} \varphi_{c}}{\partial t^{2}}-\nabla^{2} \varphi_{c}+\mathcal{V}(\vec{x}, t)=0
$$

Passing to the Hamiltonian in terms of the operators $\hat{\psi}$ and their canonical momenta $\hat{\pi}$

$$
H=\frac{1}{2} \int d^{d} x\left[\hat{\pi}^{2}+(\nabla \hat{\psi})^{2}+M^{2}(\vec{x}, t) \hat{\psi}^{2}+\mathcal{C}(\vec{x}, t)\right]
$$

the Heisenberg equations of motion for the operators become

$$
\left[\frac{\partial^{2}}{\partial t^{2}}-\nabla^{2}+M^{2}(\vec{x}, t)\right] \hat{\psi}(\vec{x}, t)=0
$$

The Hartree factorization requires the quantity

$$
G(x, x ; t)=\left\langle\hat{\psi}^{2}(\vec{x}, t)\right\rangle
$$

in which $\langle\cdots\rangle$ stands for expectation value in the initial Gaussian wave-functional or density matrix since in the Heisenberg picture the operators evolve in time but the states do not. Since $\mathcal{V}$ and $M^{2}$ are expectation values of the first and second derivative of the potential as given by eqs. (2.49,2.50) this implies a functional relation of both $\mathcal{V} ; M^{2}$ on $G(x, x ; t)$ leading to a self-consistent condition.

An alternative description of the non-equilibrium dynamics is based on the Keldysh or closed time path method 44] in which the main ingredient is the time evolution of an initial density matrix, which could describe either a pure or mixed state

$$
\hat{\rho(t)}=U\left(t, t_{0}\right) \rho\left(\hat{t}_{0}\right) U^{-1}\left(t, t_{0}\right)
$$

where $U\left(t, t_{0}\right)$ is the time evolution operator, which is completely determined by the Hamiltonian of the system, with boundary condition $U\left(t_{0}, t_{0}\right)=1$.

Non-equilibrium expectation values and correlation functions in this time evolved density matrix can be handily obtained via a path integral representation of the generating functional of non-equilibrium correlation functions along a contour in complex time 44]

$$
\left.Z\left[j^{+}, j^{-}\right]=\int \mathcal{D} \varphi^{+} \mathcal{D} \varphi^{-} e^{i \int d t d^{d} x\left\{\mathcal{L}\left[\varphi^{+}, j^{+}\right]-\mathcal{L}\left[\varphi^{-}, j^{-}\right]\right.}\right\}
$$

where the fields and sources $\varphi^{ \pm} ; j^{ \pm}$refer to the forward $(+)$and backward (-) time branches, and functional derivatives with respect to the sources generate all the non-equilibrium correlation functions. The reader is referred to the ample literature for more details 44,,28, 25, 31].

The Gaussian approximation is implemented by performing the shift of fields given by eq. (2.44) with the same $\varphi_{c}$ on both branches since it is the expectation value and with the factorization given by eqs. (2.49,2.50) for the fields on both branches. 
In this factorization scheme and after an integration by parts in the generating functional, the effective lagrangian density becomes

$$
\mathcal{L}\left[\varphi_{c}, \psi\right]=\mathcal{L}\left[\varphi_{c}\right]+\frac{1}{2}\left\{\partial_{\mu} \psi \partial^{\mu} \psi-\psi^{2} M^{2}\right\}+\psi\left\{-\partial_{\mu} \partial^{\mu} \varphi_{c}-\mathcal{V}\right\}
$$

The effective Lagrangian density necessary for the calculation of non-equilibrium correlation functions in the CTP-Keldysh scheme is given by $\mathcal{L}_{\text {eff }}\left[\varphi^{+}, \varphi^{-}\right]=\mathcal{L}\left[\varphi_{c}, \psi^{+}\right]-\mathcal{L}\left[\varphi_{c}, \psi^{-}\right]$. Thus we see that this factorization scheme leads to a Gaussian generating functional in which the correlation functions are obtained self-consistently. Examples are described in detail below. The condition (2.45) is ensured by requiring the vanishing of the term linear in $\psi$. This is the tadpole equation 31] that leads to the equation of motion for the expectation value given by (2.51).

An alternative manner to confirm the Hartree factorization given by eqs. (2.46,2.47,2.48) in this scheme is to write in the original Lagrangian density the even and odd parts of the potential after the shift (2.44) as

$$
\begin{aligned}
V^{e}\left[\varphi_{c}, \psi\right] & =V\left[\varphi_{c}\right]+\psi^{2} M^{2}(\vec{x}, t)+\delta^{e} \\
V^{o}\left[\varphi_{c}, \psi\right] & =\psi \mathcal{V}(\vec{x}, t)+\delta^{o}
\end{aligned}
$$

and treat $\delta^{e, o}$ as 'counterterms'. The Gaussian approximation is completely determined by the one and two point functions, and these counterterms are obtained by requiring that $\delta^{\circ}$ gives a vanishing contribution to the one point function and $\delta^{e}$ gives a vanishing contribution to the two point function. This requirement leads to the identifications of $\mathcal{V}$ and $M^{2}$ given in eqs. (2.49,2.50).

In the CTP formulation the important non-equilibrium Green's functions are given by the time-ordered $\mathcal{G}^{++}$, anti-time ordered $\mathcal{G}^{--}$and Wightman $\mathcal{G}^{+-}, \mathcal{G}^{-+}$functions given by

$$
\begin{aligned}
& \mathcal{G}^{++}\left(x, t ; x^{\prime}, t^{\prime}\right)=\left\langle\psi^{+}(x, t) \psi^{+}\left(x^{\prime}, t^{\prime}\right)\right\rangle=\left\langle T \psi(x, t) \psi\left(x^{\prime}, t^{\prime}\right)\right\rangle \\
& \mathcal{G}^{--}\left(x, t ; x^{\prime}, t^{\prime}\right)=\left\langle\psi^{-}(x, t) \psi^{-}\left(x^{\prime}, t^{\prime}\right)\right\rangle=\left\langle\tilde{T} \psi(x, t) \psi\left(x^{\prime}, t^{\prime}\right)\right\rangle \\
& \mathcal{G}^{+-}\left(x, t ; x^{\prime}, t^{\prime}\right)=-\left\langle\psi^{+}(x, t) \psi^{-}\left(x^{\prime}, t^{\prime}\right)\right\rangle=-\left\langle\psi\left(x^{\prime}, t^{\prime}\right) \psi(x, t)\right\rangle \\
& \mathcal{G}^{-+}\left(x, t ; x^{\prime}, t^{\prime}\right)=-\left\langle\psi^{-}(x, t) \psi^{+}\left(x^{\prime}, t^{\prime}\right)\right\rangle=-\left\langle\psi(x, t) \psi\left(x^{\prime}, t^{\prime}\right)\right\rangle
\end{aligned}
$$

With $T ; \tilde{T}$ the time and anti-time ordering operators respectively.

Since the Hartree approximation is local in space and time $\mathcal{V}$ and $M^{2}$ are local in space and time, the self-consistent dynamics only involves the spatial coincidence limit of the following Wightman function

$$
G\left(\vec{x}, \vec{x}^{\prime} ; t\right)=\left\langle\hat{\psi}(\vec{x}, t) \hat{\psi}\left(\vec{x}^{\prime}, t\right)\right\rangle=-\mathcal{G}^{+-}\left(x, t ; x^{\prime}, t\right)=-\mathcal{G}^{-+}\left(x, t ; x^{\prime}, t\right)
$$

The time evolution of this Green's function leads to a closed Hamiltonian system of equations equivalent to the method of equal time Green's functions presented above, eqns. (2.16 2.19) with the identification of the self consistent mass $M$ and "potential" $\mathcal{V}$ :

$$
\begin{aligned}
\dot{G}\left(\vec{x}, \vec{x}^{\prime} ; t\right) & =D\left(\vec{x}, \vec{x}^{\prime}, t\right)+\tilde{D}\left(\vec{x}, \vec{x}^{\prime}, t\right) \\
D\left(\vec{x}, \vec{x}^{\prime}, t\right) & =\left\langle\hat{\dot{\psi}}(\vec{x}, t) \hat{\psi}\left(\vec{x}^{\prime}, t\right)\right\rangle ; \tilde{D}\left(\vec{x}, \vec{x}^{\prime}, t\right)=\left\langle\hat{\psi}(\vec{x}, t) \hat{\dot{\psi}}\left(\vec{x}^{\prime}, t\right)\right\rangle=D\left(\vec{x}^{\prime}, \vec{x}, t\right)+i \delta^{d}\left(\vec{x}-\vec{x}^{\prime}\right)
\end{aligned}
$$




$$
\begin{aligned}
& \dot{D}\left(\vec{x}, \vec{x}^{\prime}, t\right)=K\left(\vec{x}, \vec{x}^{\prime}, t\right)+\left(\nabla_{x}^{2}-M^{2}(\vec{x}, t)\right) G\left(\vec{x}, \vec{x}^{\prime}, t\right) \\
& \dot{\tilde{D}}\left(\vec{x}, \vec{x}^{\prime}, t\right)=K\left(\vec{x}, \vec{x}^{\prime}, t\right)+\left(\nabla_{x^{\prime}}^{2}-M^{2}\left(\vec{x}^{\prime}, t\right)\right) G\left(\vec{x}, \vec{x}^{\prime}, t\right) \\
& K\left(\vec{x}, \vec{x}^{\prime}, t\right)=\left\langle\hat{\dot{\psi}}(\vec{x}, t) \hat{\dot{\psi}}\left(\vec{x}^{\prime}, t\right)\right\rangle \\
& \dot{K}\left(\vec{x}, \vec{x}^{\prime}, t\right)=\left(\nabla_{x}^{2}-M^{2}(\vec{x}, t)\right) D\left(\vec{x}, \vec{x}^{\prime}, t\right)+\left(\nabla_{x^{\prime}}^{2}-M^{2}\left(\vec{x}^{\prime}, t\right)\right) \tilde{D}\left(\vec{x}, \vec{x}^{\prime}, t\right)
\end{aligned}
$$

This system of equations is Hamiltonian and can be updated in time by providing initial Cauchy data on the three functions $G, D, K$ at an initial time as a function of two variables $\vec{x}, \vec{x}^{\prime}$. Since ultimately we only need the concidence limit to solve the self-consistent dynamical problem not all of the range of $\vec{x}^{\prime}$ is necessary to be stored numerically and for a given value of $\vec{x}$ only values of $\vec{x}^{\prime}$ in the immediate neighborhood are necessary (see discussion in section VII).

Of all the different available formulations that we presented above, we prefer the closedtime path method with the Hartree factorization leading to the evolution equations (2.652.69) as these present an economical and implementable system of update equations. However the main purpose of enumerating and analyzing the different alternatives is to provide a detailed assessment of the advantages and shortcomings of each method.

However before deciding on a numerical implementation of this system of equations two important steps are needed, the first is to provide self-consistent initial data for $G, D, K$ and second is to address the renormalization issues to guarantee that the dynamics will not depend on ultraviolet (short distance) cutoffs. We will address these two issues in definite and relevant $1+1$ and $3+1$ dimensional examples below.

\section{INITIAL VALUE PROBLEM}

In all of the methods detailed in the previous section in which a closed Hamiltonian system of equations for the Green's functions determines completely the dynamics, the first step towards a dynamical implementation is to provide the initial value problem, i.e. the Cauchy data at the initial time for the Green's functions.

The time evolution will be completely determined upon providing initial conditions on $\varphi_{c}$ and the Green's functions. The self-consistent approach requires to solve self-consistently the initial condition problem at $t=0$. Once the solution to the self-consistent initial value problem is obtained the self-consistent time evolution is obtained from the closed time path set of equations (2.65-2.69) obtained in the previous section.

Because the set of equations are self-consistent, to provide this initial data is tantamount to solve the initial value problem for the Green's functions self-consistently for an initial inhomogeneous classical field configuration $\varphi_{c}(\vec{x}, t=0)$, where we have chosen $t=0$ as the initial time.

Our strategy for setting up the initial value problem and the Cauchy data for the Green's function is more transparently described by focusing on the Heisenberg equations (2.53). At the initial time we expand the quantum fluctuation field $\psi$ and its canonical conjugate momentum $\hat{\pi}=\hat{\dot{\psi}}$ in terms of a complete set of functions as

$$
\hat{\psi}(\vec{x}, t=0)=\int \frac{d^{d} k}{\sqrt{2 \omega_{k}}}\left\{a_{\vec{k}} \psi_{k}(\vec{x})+a_{\vec{k}}^{\dagger} \psi_{k}^{*}(\vec{x})\right\}
$$




$$
\hat{\pi}(\vec{x}, t=0)=\hat{\dot{\psi}}(\vec{x}, t=0)=-i \int d^{d} k \sqrt{\frac{\omega_{k}}{2}}\left\{a_{\vec{k}} \psi_{k}(\vec{x})-a_{\vec{k}}^{\dagger} \psi_{k}^{*}(\vec{x})\right\}
$$

with $a_{\vec{k}} ; a_{\vec{k}}^{\dagger}$ canonical annihilation and destruction operators. The mode functions $\psi_{k}(\vec{x})$ are chosen to be the complete set of solutions of the following eigenvalue problem at the initial time

$$
\left[-\omega_{k}^{2}-\nabla^{2}+M^{2}(\vec{x}, t=0)\right] \psi_{k}(\vec{x})=0
$$

In terms of these modes and the expansion of the quantum field and its canonical momentum given by eq. (3.1,3.2) the Hamiltonian (2.52) at the initial time $(t=0)$ is given by

$$
H(t=0)=\int d^{d} k \omega_{k}\left(a_{k}^{\dagger} a_{k}+1 / 2\right)+\int d^{d} x \mathcal{C}(\vec{x}, t=0)
$$

Having found the complete set of mode functions, the initial values of $G\left(\vec{x}, \vec{x}^{\prime} ; t=\right.$ $0)$; $D\left(\vec{x}, \vec{x}^{\prime} ; t=0\right) ; K\left(\vec{x}, \vec{x}^{\prime}, t=0\right)$ are completely determined by determining the initial correlations $\left\langle a_{k}^{\dagger} a_{k^{\prime}}\right\rangle ;\left\langle a_{k} a_{k^{\prime}}\right\rangle$ in the initial state. The most physically reasonable choice is given by

$$
\left\langle a_{k}^{\dagger} a_{k^{\prime}}\right\rangle=N_{k} \delta_{k, k^{\prime}} ;\left\langle a_{k} a_{k^{\prime}}\right\rangle=0
$$

This choice corresponds to an initial state that is a density matrix which is diagonal in the basis of the number operator $a_{k}^{\dagger} a_{k}$ with occupation number $N_{k}$. In particular for $N_{k}=0$ the initial state is a pure state and corresponds to the Fock vacuum annihilated by the $a_{k}$ and is given by the instantaneous ground state of the Hamiltonian (2.52) at $t=0$.

At this stage we can make contact with the variational wave-functional by expanding the operator $\psi$ in the Schrödinger representation in terms of the complete set of states $\psi_{k}(\vec{x})$ at $t=0$

$$
\psi(\vec{x}, t=0)=\int d^{d} k \eta_{k} \psi_{k}(\vec{x}) \quad ; \quad \eta_{k}^{*}=\eta_{-k}
$$

where he choose the mode functions in such a way that $\psi_{k}^{*}(\vec{x})=\psi_{-k}(\vec{x})$ and the condition on the coefficients $\eta_{k}$ in (3.6) is a result of the hermiticity of the field $\psi(\vec{x})$. A pure state wavefunctional at the initial time can now be written as

$$
\begin{aligned}
\Psi[\eta] & =N e^{-\sum_{k, k^{\prime}} K_{k, k^{\prime}} \eta_{k} \eta_{k^{\prime}}+i \sum_{k} \pi_{c, k} \eta_{k}} \\
K_{k, k^{\prime}} & =\int d^{d} \vec{x} d^{d} \overrightarrow{x^{\prime}} \mathcal{K}\left(\vec{x}, \vec{x}^{\prime}\right) \psi_{k}(\vec{x}) \psi_{k^{\prime}}\left(\vec{x}^{\prime}\right) \\
\pi_{c, k} & =\int d^{d} \vec{x} \pi_{c}(\vec{x}) \psi_{k}(\vec{x})
\end{aligned}
$$

The choice leading to the initial correlations (3.5) corresponds to a wave-functional which is the ground state for all of the harmonic modes with frequencies $\omega_{k}$ and $\pi_{c}=0$, i.e.

$$
K_{k, k^{\prime}}=\frac{\omega_{k}}{2} \delta_{k,-k^{\prime}} ; \pi_{c, k}=0
$$

This choice leads to the identification of the real and imaginary parts of the kernel

$$
G_{k, k^{\prime}}^{-1}=2 \omega_{k} \delta_{k, k^{\prime}} ; \quad \Sigma_{k, k^{\prime}}=0
$$

For an analogous approach see [46]. 


\section{IV. $1+1$ DIMENSIONS: KINKS AND LUMPS}

We begin by implementing the strategy to set up the initial Cauchy data as described in the previous section in two relevant examples in $1+1$ dimensions. Not only does $1+1$ dimensions provide a simpler setting within which to gain experience and intuition, but also it is a relevant example for statistical mechanics and condensed matter physics since both SineGordon and $\phi^{4}$ theories are models for quasi-one dimensional charge density wave systems 12 14. In these systems transport phenomena is mediated by coherent and collective excitations such as solitons, breathers and pulse-like excitations known as polarons [12 14]. Furthermore there is a large body of results on these quantum field theories [47]- [51] that we can use as a yardstick against which to compare our approximations

\section{A. Sine-Gordon field theory}

The exactly integrable Sine-Gordon field theory provides a good example to test the reliability of variational methods since the quantum S-matrix is exactly known for all particles

as well as the expectation values of local operators on asymptotic states. This theory is also known to have a phase transition as the coupling constant is increased [49,52].

The Sine-Gordon field theory is described by the Hamiltonian:

$$
H=\int d x\left[\frac{1}{2} \pi(x, t)^{2}+\frac{1}{2}(\nabla \varphi(x, t))^{2}-\frac{\alpha_{0}}{\beta^{2}} \cos \beta \varphi\right]
$$

Following the steps described in detail in the previous sections, we find that the Hartree factorization for this problem leads to

$$
\begin{aligned}
\mathcal{V}(x, t) & =\frac{\alpha_{0}}{\beta} e^{-\frac{\beta^{2}}{2} G(x, x ; t)} \sin \beta \varphi_{c}(x, t) \\
M^{2}(x, t) & =\alpha_{0} e^{-\frac{\beta^{2}}{2} G(x, x ; t)} \cos \beta \varphi_{c}(x, t) \\
G(x, x ; t) & =\left\langle\hat{\psi}^{2}(x, t)\right\rangle
\end{aligned}
$$

The equations of motion that determine the dynamics in the Gaussian approximation thus become

$$
\begin{aligned}
& \frac{\partial^{2} \varphi_{c}}{\partial t^{2}}-\frac{\partial^{2} \varphi_{c}}{\partial x^{2}}+\frac{\alpha_{0}}{\beta} e^{-\frac{\beta^{2}}{2} G(x, x ; t)} \sin \beta \varphi_{c}=0 \\
& {\left[\frac{\partial^{2}}{\partial t^{2}}-\frac{\partial^{2}}{\partial x^{2}}+\alpha_{0} e^{-\frac{\beta^{2}}{2} G(x, x ; t)} \cos \beta \varphi_{c}\right] \hat{\psi}(x, t)=0}
\end{aligned}
$$

with the self-consistency condition given by eq.(4.4).

\section{1. 'Vacuum' sector:}

As mentioned above, the Sine-Gordon field theory has a phase transition at a critical value of the coupling constant 49,52]. We will now show that this Gaussian approximation 
reproduces this results of the Sine-Gordon field theory, giving us confidence in the approximation. For this we consider the vacuum sector with a homogeneous value of the expectation value $\varphi_{c} \equiv 0$ modulo $2 \pi$. In this simple case the Heisenberg equations are solved in terms of plane waves, leading to

$$
\begin{aligned}
G_{0}(x, y) & =\frac{1}{2 \pi} \int d k g_{0}(k) e^{i k(x-y)} \\
g_{0}(k) & =\frac{1}{2\left(k^{2}+\mu^{2}\right)^{1 / 2}} ; \mu^{2}=\alpha_{0} e^{-\frac{\beta^{2}}{2} G_{0}} ; G_{0}=G_{0}(x, x)
\end{aligned}
$$

In the vacuum sector we find for the expectation value of the Hamiltonian density in the Gaussian state

$$
\mathcal{H}\left(\mu^{2}\right)=\frac{1}{8 \pi} \int_{-\Lambda}^{\Lambda} d k \frac{2 k^{2}+\mu^{2}}{\left(k^{2}+\mu^{2}\right)^{1 / 2}}-\frac{\alpha_{0}}{\beta^{2}}\left(\frac{\Lambda^{2}}{\mu^{2}}\right)^{-\beta^{2} / 8 \pi}
$$

where we have introduced an upper momentum cutoff in the $k$-integral. We now define the renormalized mass parameter

$$
\alpha_{r}=\alpha_{0}\left(\frac{m^{2}}{\Lambda^{2}}\right)^{\beta^{2} / 8 \pi}
$$

with $G_{0}=\left\langle\hat{\psi}^{2}\right\rangle$ in the vacuum state and $\kappa^{2}$ an arbitrary renormalization scale. The energy density once subtracted at the renormalization scale $\kappa^{2}$ is given by

$$
\mathcal{H}\left(\mu^{2}\right)-\mathcal{H}\left(\kappa^{2}\right)=\frac{1}{8 \pi}\left(\mu^{2}-\kappa^{2}\right)-\frac{\alpha_{r}}{\beta^{2}}\left(\frac{\mu^{2}}{\kappa^{2}}\right)^{\beta^{2} / 8 \pi}
$$

Choosing the subtraction point to be

$$
\kappa^{2}=\alpha_{r}
$$

then the extremum of the energy is at

$$
\mu^{2}=\kappa^{2}
$$

Since the second derivative of the energy is given by:

$$
\frac{\partial^{2} \mathcal{H}}{\left(\partial \mu^{2}\right)^{2}}=-\frac{1}{8 \pi \kappa^{2}}\left(\frac{\beta^{2}}{8 \pi}-1\right)\left(\frac{\mu^{2}}{\kappa^{2}}\right)^{\left(\beta^{2} / 8 \pi\right)-2}
$$

we find that a stable ground state exists only for

$$
\frac{\beta^{2}}{8 \pi}<1
$$

which is in accord with the result of Coleman 49. For $\beta^{2}>8 \pi$ the Sine-Gordon theory undergoes a Kosterlitz-Thouless phase transition [52]. Whereas the theory is superrenormalizable for $\beta^{2}<8 \pi$ it becomes renormalizable at $\beta^{2}>8 \pi$ since the cosine operator becomes marginal at this value of $\beta$ [52]. The region $\beta^{2}>8 \pi$ must be studied within a double expansion in $\alpha_{r}$ and $\delta=\beta^{2}-8 \pi$ [52].

We will restrict our study of the Sine-Gordon field theory to the superrenormalizable regime i.e. $\beta^{2}<8 \pi$ since the Gaussian approximation cannot describe correctly the physics for $\beta^{2}>8 \pi$ [52]. 


\section{Initial value problem: Self-consistent Kinks and Lumps}

At the classical level, the Sine-Gordon model has moving kink solutions 47

$$
\phi(x, t)=\frac{4}{\beta} \tan ^{-1}\left\{\exp \left[\frac{\alpha_{0}}{\left(1-v^{2}\right)^{1 / 2}}\left(x-q_{0}-v t\right)\right]\right\}
$$

where $q_{0}$ is the position of the kink at time $t=0$ and $v$ its velocity. The dependence of the solution on $x, t$ reflects the translational and Lorentz invariance of the theory. The energy of this moving kink is 47,50

$$
E=\frac{8 \alpha_{0}}{\left(1-v^{2}\right)^{1 / 2}}
$$

Thus the classical (c-number) kink is a relativistically invariant model of an extended body in one spatial dimension.

For a kink solution at rest we have:

$$
\begin{aligned}
& \cos \beta \phi(x)=1-\frac{2}{\cosh ^{2} \alpha_{0} x} \\
& \sin \beta \phi(x)=\frac{2 \sinh \alpha_{0} x}{\cosh ^{2} \alpha_{0} x}
\end{aligned}
$$

We seek to set up an initial value problem that can include as self-consistent solutions kink-like configurations to be identified as a classical kink dressed by the quantum corrections. As described in a previous section, this is achieved by seeking self-consistent solutions of the following eigenvalue problem

$$
\begin{aligned}
& {\left[-\omega_{k}^{2}-\frac{d^{2}}{d x^{2}}+\mu^{2} e^{-\frac{\beta^{2}}{2} \tilde{G}(x, x)} \cos \beta \varphi_{c}(x)\right] \psi_{k}(x)=0} \\
& \tilde{G}(x, x)=G(x, x)-G_{0}
\end{aligned}
$$

where $\mu^{2} ; G_{0}$ are given by eq. (4.6) and with $\varphi_{c}$ a classical configuration with a kink-like profile to be found self-consistently. In the presence of a kink-like configuration the quantum fluctuations asymptotically far away from the kink will be similar to vacuum fluctuations since $\varphi_{c}(x) \rightarrow 2 \pi$ far away from the kink, therefore $G(x, x)-G_{0} \rightarrow 0$ as $x \rightarrow \infty$. This leads to the effective mass of the excitations to be given by $\mu$.

The spectrum of harmonic quantum fluctuations around soliton solutions of the SineGordon theory is obtained from a Schrödinger like equation with a a potential of the form

$$
v(x)=m^{2} \frac{n(n+1)}{\cosh ^{2}[m x]}
$$

where $m$ is the (asymptotic) mass of the fluctuations and for the Sine-Gordon model $n=1$. Potentials of this form are reflectionless and integrable and their spectrum is exactly known [53]. Therefore we propose a self-consistent potential for the mode functions of the form

$$
\mu^{2} e^{-\frac{\beta^{2}}{2} \tilde{G}(x, x)} \cos \beta \varphi_{c}(x) \equiv M^{2}-\frac{2 m^{2}}{\cosh ^{2}[m x]}
$$


where the parameters $M^{2} ; m^{2}$ are identified with the asymptotic mass of the quanta and the width of the kink or lump configuration and will be self-consistently determined from the parameters of the theory $\mu^{2} ; \beta$.

We want to stress that eq.(4.18) is an additional ansatz, independent of the variational approximation defined by eqs.(4.4)-(4.5). We remark that although Eqs. (4.4)-(4.5) admit much more general solutions, the simple ansatz (4.18) is introduced to facilitate an analytic self-consistent solution to the initial value problem. Eqs.(4.4)-(4.5) in general require a numerical solution (see sec. VII) which is computationally intensive.

With this ansatz the eigenvalue problem becomes

$$
\left[\frac{d^{2}}{d x^{2}}+W_{k}^{2}-m^{2}+\frac{2 m^{2}}{\cosh ^{2}[m x]}\right] \psi_{k}(x)=0 ; \quad W_{k}^{2}=w_{k}^{2}+m^{2}-M^{2}
$$

In terms of the solutions of this eigenvalue problem we will choose the initial Gaussian state to be that of the ground state for the Harmonic oscillators of frequency $\omega_{k}$.

The solutions to this eigenvalue problem are available in the literature 47,53

$$
\begin{array}{r}
\psi_{0}(x)=\sqrt{\frac{m}{2}} \frac{1}{\cosh [m x]} ; W_{k}^{2}=0 \Rightarrow \omega_{0}^{2}=M^{2}-m^{2} \\
\psi_{k}(x)=\frac{e^{i k x}}{\sqrt{k^{2}+m^{2}}}[i k-m \tanh [m x]] ; W_{k}^{2}=k^{2}+m^{2} \Rightarrow \omega_{k}^{2}=k^{2}+M^{2}
\end{array}
$$

We see that the mass of the asymptotic particle states around the classical configuration is given by $M$ allowing us to identify at once this parameter with the (renormalized) mass parameter in the Sine Gordon equation

$$
M^{2}=\mu^{2}
$$

Requiring the frequency of the bound state (4.20) to be positive restricts $M^{2}>m^{2}$, the continuum modes asymptotically are phase shifted plane waves. The Heisenberg field operators and its canonical conjugate momentum at the initial time are expanded as

$$
\begin{aligned}
& \hat{\psi}(x, t=0)=\frac{1}{\sqrt{2 \omega_{0}}}\left(a_{0}+a_{0}^{\dagger}\right) \psi_{0}(x)+\sum_{k} \frac{1}{\sqrt{2 \omega_{k}}}\left[a_{k} \psi_{k}(x)+a_{k}^{\dagger} \psi_{k}^{*}(x)\right] \\
& \hat{\pi}(x, t=0)=-i \sqrt{\frac{\omega_{0}}{2}}\left(a_{0}-a_{0}^{\dagger}\right) \psi_{0}(x)-i \sum_{k} \sqrt{\frac{\omega_{k}}{2}}\left[a_{k} \psi_{k}(x)-a_{k}^{\dagger} \psi_{k}^{*}(x)\right]
\end{aligned}
$$

As mentioned above the initial state is chosen to be the ground state for the Harmonic oscillators of frequency $\omega_{k}$, i.e. this state is the vacuum of all the annihilation operators. We find

$$
\begin{aligned}
G(x, x) & =G_{0}+\frac{F[\eta]}{\cosh ^{2}[m x]} ; \eta=\frac{m}{\sqrt{M^{2}-m^{2}}} \\
F[\eta] & =\frac{\eta}{2 \pi} \operatorname{ArcTg}[\eta]
\end{aligned}
$$

The ansatz (4.18) leads to the following consistency equations 


$$
\begin{aligned}
& M^{2}=\mu^{2} \\
& \cos \beta \varphi_{c}(x)=e^{\frac{\beta^{2} F[\eta]}{2 \cosh ^{2}[m x]}}\left[1-\frac{2 \eta^{2}}{1+\eta^{2}} \frac{1}{\cosh ^{2}[m x]}\right]
\end{aligned}
$$

The first equation is simply the statement that the asymptotic states far away from the soliton must describe quanta of mass $\mu$, which has already been argued above in eq. (4.22). For a topological kink solution we must impose that $\varphi_{c}(x \rightarrow-\infty) \rightarrow 0 ; \varphi_{c}(x \rightarrow+\infty) \rightarrow 2 \pi$. This requires that $\cos \beta \varphi_{c}(x \rightarrow \pm \infty) \rightarrow 1$ but it must cross zero twice when the argument is $\pi / 2 ; 3 \pi / 2$ and that the minimum value of the cosine is -1 . These two conditions lead to

$$
\begin{aligned}
& \eta^{2}>1 \\
& \frac{\beta^{2}}{2}=\frac{1}{F[\eta]} \ln \left[\frac{\eta^{2}+1}{\eta^{2}-1}\right]
\end{aligned}
$$

The second condition (4.29) determines $m=m(\beta)$ and for all values of $\beta$ it is constrained in the interval

$$
\frac{\mu^{2}}{2} \leq m^{2} \leq \mu^{2}
$$

this is a rather mild constraint on the width of the soliton and confirms that in the weak coupling limit $\beta \rightarrow 0 m \rightarrow \mu \approx \alpha_{0}$ as is expected. Within the region (4.30) there are solutions for arbitrary $\beta$.

This method also allows us to construct self-consistent solutions of the initial value problem for the case of non-topological lumps. For all values of the parameters (still with $\mu^{2}>m^{2}$ ) such that

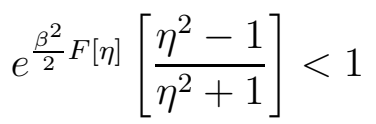

the solution provides a classical field configuration whose asymptotic value resides in the same vacuum state and is clearly an excited state that will relax into mesons under time evolution.

In this variational approximation, physical quantities, such as the soliton profile, the energy, etc, are analytic functions of $\eta^{2}$. It is then interesting to connect $\eta^{2}$ with the SG coupling constant $\beta$. In the weak coupling limit $(\beta \rightarrow 0)$, we see that $\eta^{2} \rightarrow \infty$. More precisely, eqs.(4.26) and (4.29) yield,

$$
\eta \stackrel{\beta \rightarrow 0}{=}\left(\frac{4}{\beta}\right)^{2 / 3}\left[1+\frac{2}{3 \pi}\left(\frac{\beta}{4}\right)^{2 / 3}+\mathcal{O}\left(\beta^{4 / 3}\right)\right]
$$

Therefore, the variational approximation yields for weak coupling a power series in $\eta^{-2} \sim$ $\beta^{4 / 3}$. This should be compared with the exact results which can be expressed in power series of $\beta^{2}$ (besides essential singularities at $\beta=0$ ). In other words, the variational approximation does not reproduce the analytic properties in the coupling constant since a cubic-root cut in $\beta$ is suggested and the weak coupling theory and the variational approximation cannot be simply matched in a series in $\beta$. 


\section{B. Comparison with exact results in the quantum sine-Gordon theory}

The expectation values of some interesting operators are exactly known in one-particle (and multi-particle) states 54,55]. Among them is $\cos \beta \phi$ which is just the trace of the energy-momentum tensor,

$$
T_{\mu}^{\mu}(x)=\frac{2 \alpha_{0}}{\beta^{2}}[1-\cos \beta \phi(x)] .
$$

Let us consider (quantum) one-soliton states $|\theta\rangle$ with rapidity $\theta$. That is a physical state with energy and momentum:

$$
E=M \cosh \theta \quad, \quad p=M \sinh \theta
$$

where $M$ is the physical quantum soliton mass.

The expectation value of $T_{\mu}^{\mu}(0)$ in such states can be written as,

$$
F(\theta) \equiv<\theta_{1}\left|T_{\mu}^{\mu}(0)\right| \theta_{2}>
$$

Here $\theta=\theta_{1}-\theta_{2}$.

One has the exact formula 55,

$$
F(\theta)=-\frac{M}{4 \xi} \frac{\sinh \theta}{\sinh \frac{\pi \theta}{2 \xi}} e^{I(\theta)},
$$

where

$$
\xi=\frac{\beta^{2}}{8\left(1-\frac{\beta^{2}}{8 \pi}\right)}
$$

and

$$
I(\theta)=\int_{0}^{\infty} \frac{d x}{x} \frac{\sinh \left[\frac{1}{2}(\pi-\xi) x\right] \sin ^{2}\left[\frac{1}{2} x \theta\right]}{\sinh [\pi x] \sinh \left[\frac{1}{2} x \xi\right] \cosh \left[\frac{1}{2} \pi x\right]}
$$

[Our normalization convention for the soliton states differs on a factor $M$ from ref. [55]].

The integral in eq.(4.34) yields the so-called minimal form factor [54]. It can be written as a double-infinite product of Gamma functions. In this way one explicitly sees that $F(\theta)$ is a meromorphic function of $\theta$. The closest pole of $F(\theta)$ to the real $\theta$-axis is at $\theta= \pm 2 i \xi$.

For large momentum transfer, the expectation value (4.32) exhibits Regge behavior;

$$
F(\theta) \stackrel{\theta \gg 1}{=}-\frac{t^{1-\frac{2 \pi}{\beta^{2}}}}{4 \xi M^{1-\frac{4 \pi}{\beta^{2}}}},
$$

where $t \equiv 4 M^{2} \cosh ^{2} \frac{\theta}{2}$ stands for the momentum transfer.

The variational expectation value of $\cos [\beta \phi(x)]$ [eq. (4.18)] depends on the coordinate $x$ indicating that the variational soliton state is not a momentum eigenstate but a superposition of momentum eigenstates,

$$
\left|\Psi>=\int d p f(p)\right| p=M \sinh \theta>
$$


where $\mid p>$ stands for quantum one-soliton states and $f(p)$ is some function normalized such that

$$
\int|f(p)|^{2} d p=1
$$

Therefore,

$$
\begin{aligned}
<\Psi\left|T_{\mu}^{\mu}(x)\right| \Psi> & =\int_{-\infty}^{+\infty} d p d p^{\prime} f(p)^{*} f\left(p^{\prime}\right) e^{i\left(p-p^{\prime}\right) x}<p\left|T_{\mu}^{\mu}(0)\right| p^{\prime}> \\
& =\int_{-\infty}^{+\infty} d p d p^{\prime} F\left(\theta\left(p, p^{\prime}\right)\right) e^{i\left(p-p^{\prime}\right) x} f\left(p^{\prime}\right) f(p)^{*}
\end{aligned}
$$

where $F(\theta)$ is the form factor (4.32) and $M^{2} \cosh \theta=\sqrt{M^{2}+p^{\prime 2}} \sqrt{M^{2}+p^{2}}-p p^{\prime}$.

Taking the Fourier transform of eq. (4.36) yields,

$$
\begin{aligned}
\int_{-\infty}^{+\infty} \frac{d x}{2 \pi} e^{i x p}<\Psi\left|T_{\mu}^{\mu}(x)\right| \Psi> & =\int d q f(q)^{*} f(q+p) F\left(\theta_{p, q}\right) \\
\text { where } \theta_{p, q} & =\operatorname{Argsinh} \frac{q+p}{M}-\operatorname{Argsinh} \frac{p}{M}
\end{aligned}
$$

We read from eq.(4.18) that

$$
<\Psi\left|T_{\mu}^{\mu}(x)\right| \Psi>_{v a r}=\frac{2}{\beta^{2}}\left(M^{2}-\frac{2 m^{2}}{\cosh ^{2}[m x]}\right) .
$$

Therefore,

$$
\int_{-\infty}^{+\infty} \frac{d x}{2 \pi} e^{i x p}<\Psi\left|T_{\mu}^{\mu}(x)\right| \Psi>_{v a r}=-\frac{2 p}{\beta^{2} \sinh \left[\frac{\pi p}{2 m}\right]} .
$$

We can match the exact expressions and the variational approximation in the weak coupling limit which is also a non-relativistic limit $p, p^{\prime}<<M, \theta<<1$ since the solitons becomes very massive for small $\beta$. Then, $\theta_{p, q} \approx \frac{q}{M}$ and

$$
\int_{-\infty}^{+\infty} \frac{d x}{2 \pi} e^{i x p}<\Psi\left|T_{\mu}^{\mu}(x)\right| \Psi>^{p \rightarrow 0}=\int_{-\infty}^{+\infty} \frac{d x}{2 \pi} e^{i x p}<\Psi\left|T_{\mu}^{\mu}(x)\right| \Psi>_{v a r} \stackrel{p \rightarrow 0}{=}-\frac{M}{2 \pi}
$$

where we used eqs.4.33), (4.35) and (4.37) and that $M=8 \mathrm{~m} / \beta^{2}$ for the soliton mass in the classical $\beta \rightarrow 0$ limit.

We do not see for large $\theta$ Regge behaviour in $F_{\text {var }}(\theta)$. Eq. (4.38) suggests that $F_{\text {var }}(\theta)$ decreases exponentially for large momentum transfer.

In summary, the simple self-consistent ansatz (4.18) does not reproduce the exact on-shell sine-Gordon except in the weak coupling regime.

Thus this initial configuration will undergo non-trivial time evolution to relax via meson emission to a stationary state of lowest energy compatible with the variational ansatz.

Since for weak coupling the variational initial configuration does not differ much from the exact solution, we expect slow time evolution at weak coupling with little meson production. Clearly a deeper insight into the further evolution of these variational initial configurations require a numerical study of eqs.(4.4)-(4.5) [see sec. VII]. 


\section{C. $\varphi^{4}$ Field Theory}

The $\varphi^{4}$ quantum field theory is the simplest theory that presents a symmetry breaking phase transition with relevant low-energy phenomenology in $3+1$ dimensions. It is also a model to describe kink-like excitations and the collective behavior of quasi-one dimensional charge density wave systems within the Landau-Ginzburg framework. The kink configurations in the broken symmetry phase are interpreted as domain walls between coexisting degenerate phases. We will study the $3+1$ dimensional version in the form of the linear sigma model in the next section. In this section we study the simpler $1+1$ dimensional model not only as a preliminary step towards the $3+1$ dimensional implementation but also because this is relevant in statistical mechanics and condensed matter to describe nonperturbatively the quantum dynamics of transport in quasi-one-dimensional charge density wave systems [12 14.

The Lagrangian density is given by

$$
\mathcal{L}=\frac{1}{2} \partial_{\mu} \varphi \partial^{\mu} \varphi+\frac{\mu^{2}}{2} \varphi^{2}-\frac{g}{4} \varphi^{4}
$$

Upon shifting the field by the expectation value $\varphi_{c}$, the Hartree factorization advocated in the previous section leads to the following equations of motion

$$
\begin{aligned}
& {\left[\frac{\partial^{2}}{\partial t^{2}}-\frac{\partial^{2}}{\partial x^{2}}-\mu^{2}+g \varphi_{c}^{2}+3 G(x, x ; t)\right] \varphi_{c}(x, t)=0} \\
& {\left[\frac{\partial^{2}}{\partial t^{2}}-\frac{\partial^{2}}{\partial x^{2}}-\mu^{2}+3 g \varphi_{c}^{2}+3 G(x, x ; t)\right] \hat{\psi}(x, t)=0} \\
& G(x, x ; t)=\left\langle\hat{\psi}^{2}(x, t)\right\rangle
\end{aligned}
$$

\section{The vacuum sector and the phase transition}

Before proceeding to set up the initial value problem for inhomogeneous time dependent configurations we will address the properties of the vacuum sector in the variational approximation. For this we look at space-time independent configurations of $\varphi_{c}$ and obtain the effective potential $V_{\text {eff }}\left(\varphi_{c}\right)=\langle\mathcal{H}\rangle$ with $\mathcal{H}$ the Hamiltonian density as introduced in section II. For this purpose it proves convenient to introduce the composite operator

$$
\hat{\chi}(x, t)=-\mu^{2}+3 g \hat{\varphi}^{2}(x, t)
$$

whose expectation value in the Gaussian variational state is given by

$$
\chi(x, t)=-\mu^{2}+3 g \varphi_{c}^{2}(x, t)+3 g G(x, x ; t)
$$

which is recognized as the space-time dependent effective mass of the quantum fluctuations.

For a space time constant configuration corresponding to the Fock vacuum of the annihilation operators, we find

$$
G(x, x)=\int \frac{d k}{2 \pi} \frac{1}{2 \sqrt{k^{2}+\chi}}
$$


and leads to the self-consistent gap equation

$$
\chi=-\mu^{2}+3 g \varphi_{c}^{2}+3 g \int \frac{d k}{2 \pi} \frac{1}{2 \sqrt{k^{2}+\chi}}
$$

Since the Heisenberg equations (4.40,4.41) must be finite, the logarithmic divergence in the integral is absorbed into a mass renormalization. This polynomial theory is superrenormalizable in $1+1$ dimensions and mass renormalization renders the theory finite.

The effective potential is given by

$$
V_{\text {eff }}=<V>=-\frac{1}{2} \mu^{2} \phi^{2}+\frac{g}{4} \phi^{4}-\frac{\mu^{2} G}{2}+\frac{3 g}{4} G^{2}+\frac{3 g}{2} G \phi^{2}
$$

which can be written in terms of the two variables $\varphi_{c} ; \chi$ (the composite operator effective potential [36]) in the form

$$
V_{e f f}(\phi, \chi)=\frac{1}{2} \chi \phi^{2}-\frac{g}{2} \phi^{4}-\frac{1}{6 g}\left[\frac{\chi^{2}}{2}+\mu^{2} \chi\right]+\frac{1}{2} \int \frac{d k}{2 \pi} \frac{1}{2 \omega(k)}
$$

This form of the effective potential is useful because $\partial V_{e f f}\left(\varphi_{c}, \chi\right) / \partial \chi$ gives the gap equation (4.46).

As a function of two variables we must minimize the potential with respect to both to find the vacuum state. The minimum of the potential is obtained from the condition

$$
\chi \phi-2 g \phi^{3}=0 .
$$

The value of $\phi$ at the minimum is the vacuum expectation value $v$ and the expectation value of the composite operator evaluated at this minimum is given by

$$
m_{r}^{2} \equiv \chi_{\phi=v}=2 g v^{2}
$$

The gap equation at the minimum is given by

$$
2 g v^{2}=-\mu^{2}+3 g\left[v^{2}+\int \frac{d k}{2 \pi} \frac{1}{2 \omega_{0}(k)}\right]
$$

where $\omega_{0}(k)=\left(k^{2}+m_{r}^{2}\right)^{1 / 2}$. We can use this value to subtract the gap equation for arbitrary $\varphi_{c}$, and the renormalized gap equation then becomes:

$$
\chi=2 g v^{2}+3 g\left(\phi^{2}-v^{2}\right)-\frac{3 g}{4 \pi} \log \left[\frac{\chi}{m_{r}^{2}}\right]
$$

Integrating the renormalized expression for $\frac{\partial V}{\partial \chi}$ with respect to $\chi$ gives us the renormalized effective potential which is:

$$
\begin{aligned}
V[\phi, \chi] & =\frac{\chi}{2}\left(\phi^{2}-v^{2}\right)-\frac{g}{2}\left(\phi^{4}-v^{4}\right) \\
& -\frac{1}{12 g}\left(\chi-m_{r}^{2}\right)^{2}+\frac{1}{8 \pi}\left[\chi-m_{r}^{2}+\chi \log \left(\frac{m_{r}^{2}}{\chi}\right)\right] .
\end{aligned}
$$


and we have chosen the potential to be zero at the minimum for convenience. As a function of the coupling $g$ this effective potential describes a (weak) first order phase transition as shown explicitly below.

It is convenient to now rescale everything in terms of the renormalized mass $m_{r}^{2}=2 g v^{2}$. we let

$$
g \rightarrow m_{r}^{2} g ;, \chi \rightarrow m_{r}^{2} \chi
$$

so that at the minimum we now have:

$$
\chi=1 ; \quad \phi^{2}=\frac{1}{2 g}
$$

The effective potential is now given by:

$$
\begin{aligned}
\frac{V[\phi, \chi, g]}{m_{r}^{2}} & =\frac{\chi}{2}\left(\phi^{2}-\frac{1}{2 g}\right)-\frac{g}{2}\left(\phi^{4}-\frac{1}{4 g^{2}}\right) \\
& -\frac{1}{12 g}(\chi-1)^{2}+\frac{1}{8 \pi}(\chi-1-\chi \log \chi) .
\end{aligned}
$$

This can be most easily plotted by using the relationship:

$$
\phi^{2}=\frac{1}{2 g}+\frac{1}{3 g}(\chi-1)+\frac{1}{4 \pi} \log \chi
$$

and parametrically plotting $V$ and $\phi$ as a function of $\chi$. To find the position of the phase transition, this takes place when the value of the potential at the minimum at $\phi=0$ has the same value as at the minimum at $\phi=v$ which we chose to be zero. Solving for this numerically we find that

$$
g_{c} / m_{r}^{2}=.3896 \pi=1.224
$$

To show this behavior we plot the effective potential at the three values $g / m_{r}^{2}=1, g_{c}, 2$ in Fig. 1.

For $g<g_{c}$ the potential has a double well structure with degenerate minima at $\varphi_{c}= \pm v$, as the value of the (dimensionless) coupling is increased the minima shifts abrubtly to $\phi=0$ when $g \geq g_{c}$, these results are similar to those found previously [56].

\section{Inhomogeneous Initial value problem: Kinks and Lumps}

The harmonic fluctuations around a stationary classical kink configuration are solutions of a Schrödinger-like eigenvalue problem with a potential given by eq. (4.17) for $n=2$ whose solutions are also known exactly [53,50]. The classical kink configuration corresponds to

$$
\varphi_{c}^{2}(x)=v^{2}\left[1-\frac{1}{\cosh ^{2}[m x]}\right]
$$

with $v$ the tree level vacuum expectation value. Thus we seek a self-consistent solution of the eigenvalue problem in the Gaussian variational approximation of the form 


$$
\varphi_{c}^{2}(x)=v^{2}-\frac{\alpha}{\cosh ^{2}[m x]} ;\left\langle\hat{\psi}^{2}(x)\right\rangle=\left\langle\hat{\psi}^{2}(\infty)\right\rangle+\frac{A}{\cosh ^{2}[m x]}
$$

We propose a variational ansatz similar to that of the Sine Gordon problem above

$$
-\mu^{2}+3 g\left[\varphi_{c}^{2}+G(x, x)\right]=M^{2}-\frac{2 m^{2}}{\cosh ^{2}[m x]}
$$

where the variational parameters $M ; m$ will be determined self-consistently. The logarithmic divergence in $\left\langle\psi^{2}(x)\right\rangle$ is independent of $x$ and is absorbed in mass renormalization, which is the only renormalization required in this superrenormalizable theory. Thus define the renormalized mass parameter

$$
\mu_{R}^{2}=\mu^{2}-3 g G_{0} ; G_{0}=\left\langle\hat{\psi}^{2}(\infty)\right\rangle
$$

Requiring that $\varphi_{c}(x)$ asymptotically for large $|x|$ becomes an exact solution of the space-time dependent problem leads to the constraint

$$
-\mu_{R}^{2} v+g v^{3}=0 \Rightarrow v^{2}=\frac{\mu_{R}^{2}}{g}
$$

Then the asymptotic solution of the eigenvalue problem leads to the identification

$$
M^{2}=2 \mu_{R}^{2}
$$

which is the usual relationship between the mass of the asymptotic quanta and the vacuum expectation value in the broken symmetry phase.

Since the ansatz (4.58) is similar to that in the Sine Gordon case studied previously, the results for $G(x, x)$ can be obtained from the previous case and is given by eq.(4.25) with

$$
G_{0}=\int \frac{d k}{2 \pi} \frac{1}{2 \sqrt{k^{2}+M^{2}}}
$$

Finally the expressions (4.25,4.62) with the identifications given by (4.59,4.60,4.61) and the ansatz for the expectation value (4.56) lead to the self-consistent solution

$$
\alpha=\frac{2 m^{2}}{3 g}+F[\eta]
$$

with $F[\eta]$ given in $(4.25,4.26)$. The only possibility for a topological kink profile is given by

$$
\alpha=v^{2} \Rightarrow \varphi_{c}^{2}(x)=v^{2} \tanh ^{2}[m x]
$$

Requiring that the initial configuration $\varphi_{c}(x)$ have a topological kink profile, the selfconsistency condition (4.63) leads to the following transcendental equation for $\eta$

$$
\frac{3-\eta^{2}}{3\left(1+\eta^{2}\right) F[\eta]}=\frac{g}{\mu_{R}^{2}}
$$


where we have used eqs.(4.64,4.60,4.61) and the expression for $\eta$ given in eqs. (4.25, 4.26 ), the right hand side of (4.65) is recognized as the dimensionless coupling. It is clear that there are solutions for any value of the dimensionless coupling with $0<\eta<3$.

We note that this variational form for $\varphi_{c}(x)$ is not a stationary solution of the equation of motion (4.40) but must be taken as an initial configuration that will eventually evolve in time.

This method also allows us to construct non-topological lump solutions with $\alpha<v^{2}$. These solutions correspond to initial inhomogeneous configurations that will relax via emission of mesons.

Although we have renormalized the theory at the initial time by mass renormalization, it is straightforward to prove that the same mass renormalization can be performed at all times. The main point is that the Heisenberg equations for the operator $\psi$ can be written in the form

$$
\left[\frac{\partial^{2}}{\partial t^{2}}-\frac{\partial^{2}}{\partial x^{2}}+M^{2}+\delta M^{2}(x, t)\right] \psi(x, t)=0
$$

and the mass counterterm $\delta M^{2}(x, t)$ is a localized function of space. The Green's function can be calculated in perturbation theory in $\delta M^{2}(x, t)$ in terms of Feynman diagrams with free field propagators of mass $M$ and mass insertions of $\delta M^{2}(x, t)$. The zeroth order has a logarithmic divergence which is absorbed in mass renormalization and all higher order insertions are finite as long as the theory remains superrenormalizable, which in the case of Sine-Gordon requires $\beta^{2}<8 \pi$. This is equivalent to obtaining the divergences in an operator product expansion.

\section{Variational approach vs. Collective Coordinates:}

A perturbative approach to quantization around a soliton configuration begins by obtaining the eigenmodes of the Schrödinger operator associated with the harmonic fluctuations around the classical kink [50,57]- [63]. There is an eigenmode with zero frequency associated with translational invariance [50,57]- 663] that must be treated non-perturbatively. This mode is isolated from the mode expansion and treated as a collective coordinate [50.57][63] (although see [48] for a different approach) and perturbation theory is carried out for the non-zero frequency modes, since these can be studied in a small amplitude expansion. Collective coordinate quantization is complicated by the fact that the functional basis is not cartesian [57,60,61 with complicated Jacobians and a prescription for normal ordering [58, 59,62, 63]. Although high order calculations of S-matrix elements [58,59,62, 63] and more recently of non-equilibrium properties 64 had been carried out, they represent an enormous technical feat.

In order to make contact with the collective coordinate program of quantization and the treatment of zero modes, we recognize that in the self-consistent solution for the fluctuations, the mode that would correspond to the zero frequency mode in the perturbative approach is precisely $\psi_{0}(x)$ with frequency $\omega_{0}=\sqrt{M^{2}-m^{2}}$ (see 4.20), that indeed becomes zero for $m=M$. However this value of $m$ leads to infrared divergences in our treatment (the variable $\eta$ given by eq. (1.25) diverges), which are a reflection of the infrared divergences that appear in the perturbative approach. We notice, however, that self-consistent solutions exist 
for a large range of the ratio $m / M$ as discussed above and there are no infrared pathologies associated with any value of $m$ for which solutions are available.

The apparent contradiction between the variational approach and the collective coordinate method is resolved when it is realized that i) the profile $\varphi_{c}(x)$ obtained from the self-consistent solution to the mode equations is not a stationary solution of the equation of motion including the quantum backreaction effects. ii) the zero mode in the perturbative treatment is a consequence of translational invariance, the change in the classical field profile under an infinitesimal shift of the origin is the zero mode of the fluctuation operator [50, 36, 57]- 63]. In the variational approach translational invariance is also present since the ansatz for the self-consistent potentials (4.18,4.58) could be written in terms of $x-x_{0}$ with $x_{0}$ an arbitrary point (the center of the soliton or lump) and the mode functions will be functions of $x-x_{0}$. However, unlike in the perturbative calculation in which an infinitesimal translation of the center of the classical configuration leads to a zero mode whose wave-function is $\propto d \varphi_{c}(x) / d x$, in the self-consistent approach, such an invariance requires an infinitesimal translation of $\varphi_{c}$ and the self-consistent potential which involves $G(x, x)$. Obviously the function obtained by an infinitesimal shift in $x_{0}$ from $\varphi_{c}$ is not a zero mode of the fluctuation operator, precisely because of the self-consistent potential that provides the quantum backreaction.

Thus our interpretation of the resolution of the apparent contradiction is that the quantum backreaction effects that dress the soliton or lump solution also remove the degeneracy associated with the zero mode. Although translational invariance is present, the self-consistent ansatz determines a profile centered at a particular point in space, shifting the center is tantamount to shifting the spatial dependence of all of the Green's functions and expectation values under which the expectation value of the Hamiltonian in the trial state is invariant.

Thus this self-consistent variational method based on a trial ansatz, provides a nonperturbative manner of circumventing the infrared singularities associated with translational zero modes in the perturbative approach.

\section{3+ 1 DIMENSIONS: INHOMOGENEOUS CHIRAL CONDENSATES}

One of our main motivations for studying inhomogeneous non-equilibrium problems is to provide a consistent framework to study the relaxation of high energy inhomogeneous configuration in heavy ion collisions via expansion into the vacuum. The main goal is to provide a microscopic non-equilibrium description of the dynamics leading to a hydrodynamic description and the scaling solutions. Of particular relevance is the study of the relaxation of inhomogeneous chiral condensates since this could be potential soft probes of the chiral phase transition in the form of enhanced pion distributions with distinct isospin correlations. In order to address these issues we must i) provide a model quantum field theory that incorporates the relevant low energy physics, ii) we must propose an initial state that incorporates the relevant geometry, leading to longitudinal or spherical expansion, iii) provide a consistent framework to study the microscopic dynamics.

For the first step we invoke the linear model in terms of a sigma field and an isospin triplet of pseudoscalar pions. In order to maintain the phenomenology of PCAC in the Gaussian approximation we invoke the large $N$ limit in which the sigma field and the pions 
are in the vector representation of the $O(N)$ group. For the initial inhomogeneous field configuration one should ideally propose either an inhomogeneous cylindrically symmetric field configuration in the sigma component (or possibly with an inhomogeneous component along a definite isospin direction if one wants to study a disoriented configuration) reminiscent of two pancakes or a disk configuration with an extension in the transverse direction of several fm.

The study of such a configuration including quantum effects as advocated in this work is an extremely complicated task and beyond the computational capabilities at this moment. We will be less ambitious and seek to understand the dynamics of a simpler configuration with spatial variation along only one coordinate, chosen to be the z-axis and translationally invariant in the perpendicular directions. Even this simpler scenario represents an advance in the treatment of semiclassical configurations plus backreaction. Lastly, the time evolution is to be studied with the methods detailed in the previous section. As in the simpler $1+1$ dimensional problems we begin by establishing the self-consistent initial value problem and Cauchy data on the necessary Green's functions and inhomogeneous configurations. We note that rather than invoking boost invariance that would imply specifying Cauchy data on a proper time hyper-surface as is the case in a hydrodynamical treatment, we emphasize that boost invariant hydrodynamics must arise from the full microscopic dynamics, and we set up the Cauchy data on an initial constant time slice $(t=0)$.

The Lagrangian density for the linear sigma model with an explicitly symmetry breaking term that gives a mass to the pions is given by

$$
\begin{aligned}
\mathcal{L} & =\frac{1}{2} \partial_{\mu} \vec{\Phi} \cdot \partial^{\mu} \vec{\Phi}-\frac{1}{2} \mu_{0}^{2} \vec{\Phi} \cdot \vec{\Phi}+\frac{\lambda}{8 N}(\vec{\Phi} \cdot \vec{\Phi})^{2}-h \sigma \\
\vec{\Phi} & =\left(\sigma, \pi_{1}, \ldots, \pi_{N-1}\right)
\end{aligned}
$$

The linear sigma model description of low energy pion phenomenology and PCAC corresponds to $N=4$ with parameters fit to reproduce the pion decay constant $F_{\pi}=93 \mathrm{Mev}$, the pion mass $M_{\pi} \approx 140 \mathrm{Mev}$ and the sigma mass (the value of $\sqrt{s}$ at which the phase shift in the pseudoscalar channel in $\pi-\pi$ scattering goes through $\pi / 2) M_{\sigma} \approx 600 \mathrm{Mev}$.

In order to implement the Gaussian approximation which is appropriate in the large $N$ limit we begin by performing the shift along the sigma direction

$$
\sigma(\vec{x}, t)=\varphi_{c}(\vec{x}, t) \sqrt{N}+\chi(\vec{x}, t) ;\langle\chi(\vec{x}, t)\rangle=0,
$$

To leading order the large N-limit is implemented by considering a Hartree-like factorization (neglecting $1 / \mathrm{N}$ terms)

$$
\begin{aligned}
\chi^{4} & \rightarrow 6\left\langle\chi^{2}\right\rangle \chi^{2}+\text { constant }, \\
\chi^{3} & \rightarrow 3\left\langle\chi^{2}\right\rangle \chi, \\
(\vec{\pi} \cdot \vec{\pi})^{2} & \rightarrow 2\left\langle\vec{\pi}^{2}\right\rangle \vec{\pi}^{2}-\left\langle\vec{\pi}^{2}\right\rangle^{2}+\mathcal{O}(1 / N), \\
\vec{\pi}^{2} \chi^{2} & \rightarrow\left\langle\vec{\pi}^{2}\right\rangle \chi^{2}+\vec{\pi}^{2}\left\langle\chi^{2}\right\rangle, \\
\vec{\pi}^{2} \chi & \rightarrow\left\langle\vec{\pi}^{2}\right\rangle \chi
\end{aligned}
$$

and assuming $O(N-1)$ invariance by writing 


$$
\vec{\pi}(\vec{x}, t)=\psi(\vec{x}, t) \overbrace{(1,1, \cdots, 1)}^{N-1} \Rightarrow\langle\vec{\pi} \cdot \vec{\pi}\rangle=N\left\langle\psi^{2}\right\rangle
$$

Alternatively the large $N$ expansion is systematically implemented by introducing an auxiliary field [24,25], this latter procedure allows a consistent implementation beyond the leading order. To leading order the two methods are equivalent [31]. This factorization can also be understood by implementing the tadpole method described in section II.

Thus to leading order, the large $\mathrm{N}$ approximation is similar to the Hartree variational approach described in section II. The Hartree and large N equations of motion are similar but not identical, they differ by a factor of three between the classical and quantum contributions 30.

The tadpole equation leads to the evolution equation for the expectation value

$$
\left[\frac{\partial^{2}}{\partial t^{2}}-\nabla^{2}-\mu_{0}^{2}+\frac{\lambda}{2} \varphi_{c}^{2}(\vec{x}, t)+\frac{\lambda}{2}\left\langle\psi^{2}(\vec{x}, t)\right\rangle\right] \varphi_{c}(\vec{x}, t)=h
$$

which is the mean-field equation obtained via the auxiliary field method [24,25]. After the large N-Hartree factorization and the implementation of the tadpole condition, the Hamiltonian for the field $\psi$ becomes quadratic and the Heisenberg equation for this field becomes

$$
\begin{aligned}
& {\left[\frac{\partial^{2}}{\partial t^{2}}-\nabla^{2}+M^{2}(\vec{x}, t)\right] \psi(\vec{x}, t)=0} \\
& M^{2}(\vec{x}, t)=-\mu_{0}^{2}+\frac{\lambda}{2} \varphi_{c}^{2}(\vec{x}, t)+\frac{\lambda}{2}\left\langle\psi^{2}(\vec{x}, t)\right\rangle
\end{aligned}
$$

We will propose a configuration $\varphi_{c}$ that only depends on one spatial coordinate and time, i.e. $\varphi_{c}(z, t)$. This simplification will allow us to make progress in setting up the initial value problem and to make use of the methods developed for the $1+1$ dimensional case. Thus for the initial value problem we will solve the eigenvalue problem (3.3) with the ansatz

$$
\begin{aligned}
\varphi_{c}^{2}(z) & =F_{\pi}^{2}+\frac{\alpha}{\cosh ^{2}[m z]} \\
M^{2}(\vec{x}) & =-\mu_{0}^{2}+\frac{\lambda}{2} \varphi_{c}^{2}(\vec{x}, t=0)+\frac{\lambda}{2}\left\langle\psi^{2}(\vec{x}, t=0)\right\rangle=M_{\pi}^{2}-\frac{2 m^{2}}{\cosh ^{2}[m z]}
\end{aligned}
$$

Asymptotically the solutions should be phase shifted plane waves describing asymptotic pions quanta of mass $M_{\pi}$ and therefore asymptotically for $x>>1 / m$ the PCAC relation $M_{\pi}^{2} F_{\pi}=h$ is fullfiled with

$$
M_{\pi}^{2}=-\mu_{0}^{2}+\frac{\lambda}{2} F_{\pi}^{2}+\frac{\lambda}{2}\left\langle\psi^{2}(\infty)\right\rangle
$$

is the renormalized pion mass. Translational invariance in the perpendicular directions allows us to write the mode functions solutions of the eigenvalue problem as 


$$
\begin{aligned}
& \psi_{k}(\vec{x})=e^{i \vec{k}_{\perp} \cdot \vec{x}_{\perp}} \psi_{k}(z) \\
& {\left[\frac{d^{2}}{d z^{2}}+W_{k}^{2}-m^{2}+\frac{2 m^{2}}{\cosh [m z]}\right] \psi_{k}(z)=0} \\
& W_{k}^{2}=\omega_{k}^{2}-\vec{k}_{\perp}^{2}-M_{\pi}^{2}+m^{2}
\end{aligned}
$$

We will take as initial state a Gaussian wavefunctional corresponding to the ground state of the harmonic oscillators with frequencies $\omega_{k}$.

In terms of the frequencies $W_{k}^{2}$ the problem is now exactly the same as the $1+1 \mathrm{di}$ mensional problems that were solved self-consistently in the previous section leading to the eigenfunctions and frequencies given by

$$
\begin{aligned}
& \psi_{0}(\vec{x})=e^{i \vec{k}_{\perp} \cdot \vec{x}_{\perp}} \sqrt{\frac{m}{2}} \frac{1}{\cosh [m z]} ; \omega_{k}^{0}=\sqrt{\vec{k}_{\perp}^{2}+M_{\pi}^{2}-m^{2}} \\
& \psi_{k}(\vec{x})=\frac{e^{i \vec{k} \cdot \vec{x}}}{\sqrt{k_{z}^{2}+m^{2}}}\left[i k_{z}-m \tanh [m z]\right] ; \omega_{k}=\sqrt{\vec{k}^{2}+M_{\pi}^{2}}
\end{aligned}
$$

where again stability requires that $M_{\pi}^{2}>m^{2}$. In the perturbative quantization approach, the transverse modes associated with $\psi_{0}(\vec{x})$ are the 'capillary' waves associated with massless fluctuations transverse to the interface with dispersion relation $\omega_{k}^{0}=|\vec{k}|$ as a consequence of the translational zero mode in the z-direction.

At the initial time the Heisenberg field operator is expanded in terms of this complete set of mode functions. With a continuum normalization

$$
\begin{aligned}
& \psi(\vec{x})=\int \frac{d^{3} k}{(2 \pi)^{3 / 2}} \frac{1}{\sqrt{2 \omega_{k}}}\left\{a(\vec{k}) \psi_{k}(\vec{x})+h . c .\right\}+\int \frac{d^{2} \vec{k}_{\perp}}{(2 \pi)} \frac{1}{\sqrt{2 \omega_{k}^{0}}}\left\{a_{0}(\vec{k}) \psi_{0}(\vec{x})+\text { h.c. }\right\} \\
& \pi(\vec{x})=-i \int \frac{d^{3} k}{(2 \pi)^{3 / 2}} \sqrt{\frac{\omega_{k}}{2}}\left\{a(\vec{k}) \psi_{k}(\vec{x})-\text { h.c. }\right\}-i \int \frac{d^{2} \vec{k}_{\perp}}{(2 \pi)} \sqrt{\frac{\omega_{k}^{0}}{2}}\left\{a_{0}(\vec{k}) \psi_{0}(\vec{x})-\text { h.c. }\right\}
\end{aligned}
$$

Taking the initial state to be the vacuum for all the annihilation operators we finally find

$$
\begin{aligned}
& \left\langle\psi^{2}(\vec{x})\right\rangle=\left\langle\psi^{2}(\infty)\right\rangle+I \\
& \left\langle\psi^{2}(\infty)\right\rangle=\int \frac{d^{3} k}{(2 \pi)^{3}} \frac{1}{2 \sqrt{\vec{k}^{2}+M_{\pi}^{2}}} \\
& I=\frac{m^{2}}{\cosh ^{2}[m z]}\left[\frac{1}{2 m} \int \frac{d^{2} \vec{k}_{\perp}}{(2 \pi)^{2}} \frac{1}{2 \sqrt{\vec{k}_{\perp}^{2}+M_{\pi}^{2}-m^{2}}}-\int \frac{d^{3} k}{(2 \pi)^{3}} \frac{1}{2 \sqrt{\vec{k}^{2}+M_{\pi}^{2}}} \frac{1}{k_{z}^{2}+m^{2}}\right]
\end{aligned}
$$

The first term is the usual coincidence limit of the two point function and has a quadratic and logarithmic divergence which as usual is absorbed in a mass renormalization. Introducing an ultraviolet cutoff the integrals in I yield to

$$
\begin{aligned}
I & =\frac{m^{2}}{\cosh ^{2}[m z]}\left\{\frac{1}{4 \pi^{2}} \ln \left[\frac{\Lambda}{M_{\pi}}\right]+H\left[\frac{M_{\pi}}{m}\right]\right\}=\frac{m^{2}}{\cosh ^{2}[m z]} \tilde{I} \\
H[x] & =\frac{1}{4 \pi^{2}}\left\{1-\sqrt{x^{2}-1} \operatorname{ArcTg} \frac{1}{\sqrt{x^{2}-1}}\right\}
\end{aligned}
$$


Finally the ansatz (5.12,5.13) leads to the following self-consistent condition for the effective mass term in the mode equation

$$
-\frac{2 m^{2}}{\cosh ^{2}[m z]}=\frac{\lambda m^{2}}{2 \cosh ^{2}[m z]}[\alpha+\tilde{I}]
$$

The logarithmic divergence is absorbed in coupling constant renormalization

$$
\frac{1}{\lambda_{R}}=\frac{1}{\lambda}+\frac{1}{16 \pi^{2}} \ln \left[\frac{\Lambda}{M_{\pi}}\right]
$$

where we chose to renormalize at the scale $M_{\pi}$. After renormalization, the self-consistent solution for the inhomogeneous expectation value becomes

$$
\varphi_{c}^{2}(z)=F_{\pi}^{2}\left[1-\left(\frac{4}{\lambda_{R}}+H\left[\frac{M_{\pi}}{m}\right]\right) \frac{m^{2}}{F_{\pi}^{2} \cosh ^{2}[m z]}\right]
$$

This self-consistent solution for the initial value problem is not a stationary solution of the evolution equation for the expectation value. This field profile will evolve in time and dissipate into meson excitations. The dynamics of this process of relaxation is studied within the time dependent scheme described above and addressed in detail below.

The mass renormalization and coupling constant renormalization make finite the effective space dependent mass term $M^{2}(\vec{x}, t=0)$, we now show that this renormalization renders the effective mass term finite self-consistently at all times. The proof is an implementation of the operator product expansion and goes as follows. Since the inhomogeneity is localized in space and asymptotically the expectation value $\varphi_{c}(\vec{x}, t)$ reaches its vacuum value, we can write

$$
M^{2}(\vec{x}, t)=M_{\pi}^{2}+\delta M^{2}(\vec{x}, t)
$$

The renormalization is obtained by requiring that the equations of motion are finite which results in the requirement that the effective mass term $M^{2}(\vec{x}, t)$ be finite for all values of the space time arguments. Since $M_{\pi}^{2}$ is the renormalized pion mass, renormalizability implies that

$$
\delta M^{2}(\vec{x}, t)=\frac{\lambda}{2}\left[G(\vec{x}, \vec{x}, t)-G_{0}+\varphi_{c}^{2}(\vec{x}, t)-\varphi_{c}^{2}(\infty)\right]=\frac{\lambda_{R}}{2}\left[G_{R}(\vec{x}, \vec{x}, t)+\varphi_{c}^{2}(\vec{x}, t)-\varphi_{c}^{2}(\infty)\right]
$$

is finite.

The divergences in $G(\vec{x}, \vec{x}, t)$ can be obtained by implementing the operator product expansion or alternatively by treating the term $\delta M^{2}(\vec{x}, t)$ as a localized perturbation, i.e. as a mass insertion in the Green's function.

It is a straightforward exercise to expand the Green's function $G(\vec{x}, \vec{x}, t)$ which is the coincidence limit of the Feynman propagator in a perturbative Feynman series. This is a tadpole diagram (a two-point function in the coincidence limit) in the presence of a background mass insertion, the zeroth order (one loop) is simply $G_{0}=\left\langle\psi^{2}(\infty)\right\rangle$ given by eq. (5.23) and which has been absorbed in the mass renormalization and is cancelled by the 
regular (space-time independent) mass counterterm $G_{0}$ in (5.30). The first order in $\delta M$ to one loop is simply a mass insertion in the tadpole that has exactly the divergence of the one-loop contribution to coupling constant renormalization (actually the renormalization of the composite operator $\left\langle\psi^{2}\right\rangle$ which is equivalent to coupling renormalization in the large $N$ limit). Since the divergence is independent of the momentum transfer into the loop and higher order insertions are finite by power counting we find the result

$$
G(\vec{x}, \vec{x}, t)-G_{0}=-\frac{1}{8 \pi^{2}} \ln \left[\frac{\Lambda}{M_{\pi}}\right] \delta M^{2}(\vec{x}, t)+\tilde{G}_{R}(\vec{x}, \vec{x}, t)
$$

with $\tilde{G}_{R}(\vec{x}, \vec{x}, t)$ being finite. This expression leads at once to the renormalization of coupling and mass just as at $t=0$. Writing

$$
\begin{aligned}
\lambda & =Z_{\lambda} \lambda_{R} \\
Z_{\lambda} & =\left[1-\frac{\lambda_{R}}{16 \pi^{2}} \ln \left[\frac{\Lambda}{M_{\pi}}\right]\right]^{-1}
\end{aligned}
$$

then the quantity

$$
\delta M^{2}(\vec{x}, t)=\frac{\lambda_{R}}{2} Z_{\lambda}\left[G(\vec{x}, \vec{x}, t)-G_{0}+\varphi_{c}^{2}(\vec{x}, t)-\varphi_{c}^{2}(\infty)\right]
$$

is finite. A more elegant and systematic treatment of the renormalization along the lines described above can be offered following the analysis presented in reference [65]. The linear sigma model description of low energy pion phenomenology dictates that in order to reproduce the values of $F_{\pi} \approx 93 \mathrm{Mev}, M_{\pi} \approx 140 \mathrm{Mev}$ and the sigma mass $\approx 600 \mathrm{Mev}$, the value of the quartic coupling is extremely large $\lambda_{R} \approx 36$. For such a large value of the coupling there is a Landau pole at a value of the cutoff $\Lambda \leq 2-3$ Gev requiring that this theory be understood as a low energy theory for scales well below this cutoff.

In a weakly coupled theory the form of the initial self-consistent configuration (5.28) would allow to prepare initial inhomogeneous states for which $\varphi_{c}$ probes the false vacuum $\varphi_{c} \approx 0$ on length scales much larger than the Compton wavelength of the pion. This would be achieved by specifying a value $m<<M_{\pi}$ such that $4 m^{2} / \lambda_{R} F_{\pi}^{2} \approx 1$. However in the strongly coupled linear sigma model and in the stable region for values of $m<M_{\pi}$ there is no range of the variational parameter $m$ that would allow to probe the 'false vacuum' region $\varphi_{c} \approx 0$. The main reason for this constraint is that we restricted our study to the stable region for which $M_{\pi}^{2}>m^{2}$ to avoid the instabilities associated with the bound state solutions given by eq. (5.19).

\section{Spinodal Instabilities from Klein's paradox}

The nature of the instabilities for $m^{2}>M_{\pi}^{2}$ is clear from the eigenvalue eq. (5.17) when written in the following form

$$
\left[-\frac{d^{2}}{d z^{2}}+\vec{k}_{\perp}^{2}+M_{\pi}^{2}-\frac{2 m^{2}}{\cosh [m z]}\right] \psi_{k}(z)=\omega_{k}^{2} \psi_{k}(z)
$$


For $m^{2}>M_{\pi}^{2}$ the potential penetrates the negative energy continuum in a region in space of width $\Delta z \approx 1 / m$ and there will be a band of perpendicular momenta $k_{\perp}$ for which there will be instabilities arising from particle production. This situation is similar to that of the 'Klein paradox' for free quantum fields in the presence of external potentials. In a region near the origin the potential is negative and strong enough to cross the threshold $2 M_{\pi}$ and produces particles. This instability towards particle production for the case $m^{2}>M_{\pi}^{2}$ is also encoded in the typical square root in the expressions (5.25).

For this case there is a band of wavectors $k_{\perp}$ for which there will be strong production of pions with soft perpendicular momentum. This mechanism of particle production is very similar to that of spinodal instabilities in the case of phase transitions, since the modes of the quantum field feel an inverted harmonic oscillator potential, i.e. an effective negative mass squared. We can deal with this interesting situation in the same manner as in previous treatment of the dynamics as an initial value problem in phase transitions with spinodal instabilities.

The method consists of preparing the initial state wave functional for the unstable modes that is the ground state wave functional for upright harmonic oscillators, with reference frequencies $\tilde{\omega}_{k}^{0}=\sqrt{\vec{k}_{\perp}^{2}+\mu^{2}}$, with $\mu^{2}$ an arbitrary positive mass parameter. This is the quantum field theory analog of preparing an initial Gaussian state, which is the ground state of a particular harmonic oscillator potential and evolving it in an inverted harmonic oscillator potential, which is the physical situation for spinodal instabilities.

Therefore we consider the situation with $m^{2}>M_{\pi}^{2}$ by expanding the field in terms of the eigensolutions $\psi_{k}$ of (5.17) as in (5.21) but with the frequencies

$$
\begin{aligned}
\Omega_{k}^{0} & =\sqrt{\vec{k}_{\perp}^{2}+\mu^{2}} \text { for } \quad \vec{k}_{\perp}^{2}<\Delta^{2} \\
\omega_{k}^{0} & =\sqrt{\vec{k}_{\perp}^{2}-\Delta^{2}} \text { for } \quad \vec{k}_{\perp}^{2}>\Delta^{2} \\
\Delta^{2} & =m^{2}-M_{\pi}^{2}>0
\end{aligned}
$$

and the initial state is annihilated by all of the annihilation operators. In this case we find the result

$$
\begin{aligned}
\left\langle\psi^{2}(\vec{x}, t=0)\right\rangle & =\left\langle\psi^{2}(\infty)\right\rangle+\tilde{I} \\
\tilde{I} & =\frac{1}{4 \pi} \ln \left[\frac{\Lambda}{M_{\pi}}\right]+F\left[\frac{M_{\pi}}{m}, \frac{\mu}{m}\right] \\
F[x, y] & =\frac{1}{8 \pi}\left[\sqrt{1+y^{2}-x^{2}}-y\right]+\frac{1}{4 \pi^{2}}\left[1+\frac{1}{2} \sqrt{1-x^{2}} \ln \frac{1-\sqrt{1-x^{2}}}{1+\sqrt{1-x^{2}}}\right]
\end{aligned}
$$

with $\left\langle\psi^{2}(\infty)\right\rangle$ given by (5.23).

The square roots and the logarithmic branch singularities are the hallmark of thresholds to particle production and provide an intuitive explanation for the spinodal instabilities for large amplitudes: the field profile probes the negative energy continuum in a localized region in space, resulting in particle production.

Renormalization proceeds as in the previous case, and leads to the final form of the self-consistent field profile for the expectation value in the case in which $m^{2}>M_{\pi}^{2}$

$$
\varphi_{c}^{2}(z)=F_{\pi}^{2}\left[1-\left(\frac{4}{\lambda_{R}}+F\left[\frac{\mu}{m}, \frac{M_{\pi}}{m}\right]\right) \frac{m^{2}}{F_{\pi}^{2} \cosh ^{2}[m z]}\right]
$$


In this case by varying $\mu ; m$ we can provide an initial self-consistent inhomogenous state that probes field configurations with large amplitudes away from the vacuum $\varphi_{c}=F_{\pi}$ in either direction.

The band of unstable perpendicular wave-vectors will result in profuse production of soft pions through the spinodal instabilities with a likely enhancement of pions in the low $p_{T}$ region. Although these spinodal instabilities could also be present in the one dimensional problems studied in the previous section, in that case there is only one mode that is unstable. In addition, integrability forbids particle production in $1+1$ dimensional sine-Gordon field theory. Physically the instability of one mode is not very interesting in the one-dimensional models where we are interested in the dynamics of soliton states. However it is certainly interesting and physically motivated in the sigma model in three spatial dimensions where we are interested in the dynamics of pion production from a coherent initial state. Furthermore, these spinodal instabilities associated with the Klein paradox phenomenon are not particular to the trial profiles studied here but will necessarily be a robust feature of inhomogeneous states for which large amplitudes of the order parameter can probe the negative energy continuum within a localized region in space, resulting in particle production.

Fig. 2 shows the profile for $\varphi_{c}(z) / F_{\pi}$ for a set of parameters that allows to probe near the false vacuum near the origin, and fig. 3 shows $\varphi_{c}(z) / F_{\pi}$ for a different set of parameters that probes a region with large amplitudes away from the vacuum. Both configurations probe large amplitude field configurations away from the vacuum in a localized region in space of width $\Delta z \approx 1 / m$.

\section{DYNAMICAL EVOLUTION: SUMMARY OF RENORMALIZED UPDATE EQUATIONS AND INITIAL CONDITIONS}

Having provided the initial state which furnishes the Cauchy data for the proper Green's functions and addressed the renormalization issues we are now in position to provide the update algorithm to study the dynamical evolution. We begin by gathering all of the equations of motion and initial conditions for $\varphi_{c}(\vec{x}, t)$ and the Green's functions which are given by eqs. (2.51,2.53,2.65-2.69)

$$
\begin{aligned}
& \dot{\varphi}_{c}(\vec{x}, t)=\pi_{c}(\vec{x}, t) \\
& \dot{\pi}_{c}(\vec{x}, t)=\nabla^{2} \varphi_{c}-\mathcal{V}\left(\varphi_{c}, G\right) \\
& \dot{G}\left(\vec{x}, \vec{x}^{\prime} ; t, t\right)=D\left(\vec{x}, \vec{x}^{\prime}, t\right)+\tilde{D}\left(\vec{x}, \vec{x}^{\prime}, t\right) \\
& \dot{D}\left(\vec{x}, \vec{x}^{\prime}, t\right)+\dot{\tilde{D}}\left(\vec{x}, \vec{x}^{\prime}, t\right)=2 K\left(\vec{x}, \vec{x}^{\prime}, t\right)+\left(\nabla_{x}^{2}+\nabla_{x^{\prime}}^{2}-M^{2}(\vec{x}, t)-M^{2}\left(\vec{x}^{\prime}, t\right)\right) G\left(\vec{x}, \vec{x}^{\prime}, t\right) \\
& \dot{K}\left(\vec{x}, \vec{x}^{\prime}, t\right)=\left(\nabla_{x}^{2}-M^{2}(\vec{x}, t)\right) D\left(\vec{x}, \vec{x}^{\prime}, t\right)+\left(\nabla_{x^{\prime}}^{2}-M^{2}\left(\vec{x}^{\prime}, t\right)\right) \tilde{D}\left(\vec{x}, \vec{x}^{\prime}, t\right)
\end{aligned}
$$

where we have made explicit that $\mathcal{V}$ depends on $\varphi_{c}(\vec{x}, t)$ and $G(\vec{x}, \vec{x}, t)$ and is given by the appropriate expressions in the $1+1$ dimensional cases (see eqns. (4.2, 4.3,4.40,4.41)) or by $M^{2}(\vec{x}, t)=M^{2}\left(\varphi_{c}, G\right)$ in the $3+1$ dimensional case in the large $N$ limit (see eqn. (5.11)). The initial conditions chosen for the dynamics of the expectation value are given by

$$
\varphi_{c}(\vec{x}, t=0)=\varphi_{c, s c}(\vec{x}) ; \pi_{c}(\vec{x}, t=0)=0
$$


where $\varphi_{c, s c}(\vec{x})$ is the self-consistent profile given by eqs. (4.27) and (4.29) for a topological profile or (4.31) for a lump profile in the Sine-Gordon case, or the profile given in eq. (4.57) with (4.63) for the $\phi^{4}$ theory in $1+1$ dimensions. In the $3+1$ dimensional sigma model in the large $\mathrm{N}$, the profile for the expectation value is given by eq. (5.28) in the stable case, i.e. without spinodal instabilities and by eq. (5.42) in the spinodally unstable case.

In order to update the equations we need to obtain the value of the kernels $D, \tilde{D}, K$ at the initial time $t=0$. This is achieved by using the Heisenberg equations of motion (2.53), the field expansion given by (3.1, 3.2) in terms of the eigenmodes $\psi_{k}$ of the differential eq. (3.3) and the initial correlations (3.5). We must distinguish between the different cases without and with spinodal instabilities.

\section{A. Without spinodal instabilities}

In this case we find the following expressions for the kernels at the initial time:

$$
\begin{aligned}
& G\left(z, z^{\prime}, t=0\right)=\frac{\psi_{0}(z) \psi_{0}\left(z^{\prime}\right)}{2 \omega_{0}}+\int \frac{d k}{2 \pi} \frac{\psi_{k}(z) \psi_{k}^{*}\left(z^{\prime}\right)}{2 \omega_{k}} \quad \text { in } 1+1 \text { dimensions } \\
& G\left(\vec{x}, \vec{x}^{\prime}, t=0\right)=\int \frac{d^{2} k_{\perp}}{(2 \pi)^{2}} \frac{\psi_{k}(\vec{x}) \psi_{k}^{*}\left(\vec{x}^{\prime}\right)}{2 \omega_{k}^{0}}+\int \frac{d^{3} k}{(2 \pi)^{3}} \frac{\psi_{k}(\vec{x}) \psi_{k}^{*}\left(\vec{x}^{\prime}\right)}{2 \omega_{k}} \text { in } 3+1 \text { dimensions }
\end{aligned}
$$

Since the eigenmodes are rather simple in form the integrals above can be carried out analytically in all cases, thus providing the initial Cauchy data for the Green's function.

The initial data for the other kernels is determined by

$$
\begin{aligned}
& D\left(\vec{x}, \vec{x}^{\prime}, t=0\right)=-\frac{i}{2} \sum_{k} \psi_{k}(\vec{x}) \psi_{k}^{*}\left(\vec{x}^{\prime}\right)=-\frac{i}{2} \delta^{2}\left(\vec{x}-\vec{x}^{\prime}\right) \\
& \tilde{D}\left(\vec{x}, \vec{x}^{\prime}, t=0\right)=\frac{i}{2} \sum_{k} \psi_{k}(\vec{x}) \psi_{k}^{*}\left(\vec{x}^{\prime}\right)=\frac{i}{2} \delta^{2}\left(\vec{x}-\vec{x}^{\prime}\right) \\
& K\left(\vec{x}, \vec{x}^{\prime}, t=0\right)=\sum_{k} \frac{\omega_{k}}{2} \psi_{k}(\vec{x}) \psi_{k}^{*}\left(\vec{x}^{\prime}\right) \\
& {\left[\nabla_{x}^{2}-M^{2}(\vec{x}, t=0)\right] G\left(\vec{x}, \vec{x}^{\prime}, t=0\right)=\left[\nabla_{x^{\prime}}^{2}-M^{2}\left(\vec{x}^{\prime}, t=0\right)\right] G\left(\vec{x}, \vec{x}^{\prime}, t=0\right)=} \\
& -\sum_{k} \frac{\omega_{k}}{2} \psi_{k}(\vec{x}) \psi_{k}^{*}\left(\vec{x}^{\prime}\right)
\end{aligned}
$$

We see that whereas the individual kernels evaluated at the initial time are divergent, the combination of the kernels that enter in the update equations above are finite and lead to the following result for the initial value problem

$$
\begin{aligned}
& \dot{G}\left(\vec{x}, \vec{x}^{\prime}, t=0\right)=0 \\
& \dot{D}\left(\vec{x}, \vec{x}^{\prime}, t=0\right)=\dot{\tilde{D}}\left(\vec{x}, \vec{x}^{\prime}, t=0\right)=0 \\
& \dot{K}\left(\vec{x}, \vec{x}^{\prime}, t=0\right)=0
\end{aligned}
$$

If the expectation value $\varphi_{c}(\vec{x})$ were a solution of the time independent evolution equation for the expectation value, we would have found an equilibrium self-consistent solution of the quantum back-reaction problem. However, as we pointed out before, the self-consistent 
profile for $\varphi_{c}(\vec{x})$ is not a static solution of the evolution equation and in the second step of the update, $\varphi_{c}(\vec{x})$ will begin to evolve and with it the effective mass squared $M^{2}(\vec{x})$ for the quantum fluctuations.

\section{B. With spinodal instabilities in $3+1$ dimensions:}

In this case case the Heisenberg field expansion is as given in eq.(5.21) but with the frequencies $\omega_{k}^{0}$ replaced by the frequencies given by eq. (5.36-5.38). In this case we find the same expression for either the $1+1$ or $3+1$ dimensional cases where the frequencies $\omega_{k}^{0}$ in the first term in eq. (6.8) are given by eqs.(5.36-5.38).

$$
\begin{aligned}
& \dot{G}\left(\vec{x}, \vec{x}^{\prime}, t=0\right)=0 \\
& \dot{D}\left(\vec{x}, \vec{x}^{\prime}, t=0\right)+\dot{\tilde{D}}\left(\vec{x}, \vec{x}^{\prime}, t=0\right)=\int \frac{d^{2} k_{\perp}}{(2 \pi)^{2}} \Theta\left(\Delta^{2}-\vec{k}_{\perp}^{2}\right) \frac{\mu^{2}+m^{2}-M_{\pi}^{2}}{\Omega_{k}^{0}} \psi_{0}(\vec{x}) \psi_{0}^{*}\left(\vec{x}^{\prime}\right) \\
& \dot{K}\left(\vec{x}, \vec{x}^{\prime}, t=0\right)=0 .
\end{aligned}
$$

Obviously the nonvanishing and positive expression (6.17) evaluated at $\vec{x}=\vec{x}^{\prime}$ which corresponds to the second derivative of $G$ with respect to time is a manifestation of the early exponential growth of the quantum fluctuations associated with the spinodal instabilities and particle production.

The systems of update equations shown above displays explicitly that although the individual kernels $K, D$ have short distance singularities, these cancel in the combinations that are necessary for the update equations. Insofar as the Green's function $G\left(\vec{x}, \vec{x}^{\prime}, t\right)$ is evaluated at separate spatial points it remains finite throughout the evolution since the initial value is finite and the update equations are in terms of finite quantities. However, once the coincidence limit is taken, quadratic and logarithmic divergences will appear in the short distance operator product expansion. From the time evolved value of $G\left(\vec{x}, \vec{x}^{\prime}, t\right)$ we must now take the coincidence limit to construct the updated effective mass $M^{2}(\vec{x}, t)$ and the short distance divergences must be renormalized. However our previous analysis of the short distance divergences determines unambiguously the manner to obtain the fully renormalized equations. In $1+1$ dimensions the equations of motion are rendered finite by mass renormalization and the replacement

$$
G(z, z) \rightarrow=G_{\text {sub }}(z, z)=G(z, z)-\int_{0}^{\Lambda} \frac{d k}{2 \pi} \frac{1}{\sqrt{k^{2}+M^{2}}}
$$

where $\Lambda$ is the upper momentum cutoff. In three dimensions we can take the spatial coincidence limit of the Green's function in the finite quantity $M_{\pi}^{2}+\delta M^{2}(\vec{x}, t)$ with $\delta M^{2}(\vec{x}, t)$ given by the expression (5.34).

\section{NUMERICAL STRATEGY:}

Although implementing a full numerical study of the evolution equations with the selfconsistent initial data is beyond the scope of this paper, we briefly describe how we will 
implement numerically the evolution in a forthcoming study. The implementation is obviously much simpler in the one dimensional case: one begins by discretizing space introducing a lattice cutoff $a$ and the upper momentum cutoff becomes $\Lambda=\pi / a$, so that the integral for $G_{0}$ can now be done at once leading to function of $M a$. Since eventually we are interested in the coincidence limit of $G\left(z, z^{\prime}\right)$ we do not need to update the functions of two variables $z, z^{\prime}$ for the whole range of the two variables, the non-locality of the update equations are mild only requiring the derivatives at separate points. Thus for fixed $z$ we can simply update the equations for $z^{\prime}$ just a few lattice spacings away in such a way that the discretized derivative does not introduce coincidences. The coincidence limit will correspond to writing $z^{\prime} \rightarrow z+a$ and the renormalized Green's function is obtained by subtracting the value of $G_{0}$, in the limit when $M a<<1$ the renormalized Green's function is insensitive to the short distance cutoff.

In $3+1$ dimensions the strategy consists of the discretization of space in a cubic lattice of equal spacing $a$ along all the directions. Now the second derivatives needed in the update equations require storing the field configurations on 6 sites around the particular points $\vec{x}, \vec{x}^{\prime}$, again since ultimately we are interested in local field configurations and the spatial coincidence limit we do not need to store the configurations for all of the range of $\vec{x}^{\prime}$ but only in the immediate neighborhood of the point $\vec{x}$. For a lattice spacing $a$ there corresponds an upper momemtum $\vec{k}_{\text {max }}=(\pi / a, \pi / a, \pi / a)$ and the momentum integrals needed for $G_{0}$ are done with spherical symmetry and a radial upper momentum cutoff $\Lambda=\sqrt{3} \pi / a$ which is the radius of the spherical Brillouin zone. The coincidence limit must be understood now as $G(\vec{x}, \vec{x}+\vec{a})$ and for $M a<<1$ the renormalized quantity $M^{2}(\vec{x}, t)$ is insensitive to the short distance cutoff.

As discussed in the previous section within the context of renormalization, the linear sigma model is a low energy theory with a cutoff $\Lambda \leq 2-3 \mathrm{Gev}$ and this restricts the value of the lattice spacing for a numerical analysis. There is, however, a much more stringent constraint on the sigma model description of low energy physics: this model does not include other important hadronic degrees of freedom such as the pseudoscalars $\eta ; \eta^{\prime}$ with masses $\approx 500-600 \mathrm{Mev}$ and vector mesons (which determine pion electromagnetic form factors) with masses $\approx 700-800 \mathrm{Mev}$. These constraints suggest that the actual physical cutoff for the sigma model is not much bigger than $\Lambda \approx 500-700 \mathrm{Mev}$ resulting in a lattice spacing such that $M_{\pi} a \approx 0.2$. Thus the combination of Landau pole arguments and low energy constraints on the dynamics require that a physically reasonable lattice spacing for a numerical treatment be of the order of $\approx 0.2 \mathrm{fm}$, which will not result in strong short distance effects in the update of the dynamical equations.

\section{CONCLUSIONS:}

In this article we focused on establishing a consistent framework to study the nonequilibrium evolution of inhomogeneous non-perturbative field configurations including quantum backreaction effects. The main goal is to provide numerically implementable schemes to study the time evolution of large amplitude field configurations including the quantum backreaction associated with particle production on the relaxational dynamics.

We proposed a self-consistent variational approach to study the non-perturbative aspects of the evolution of large amplitude inhomogeneous configuration, and analyzed the avail- 
able frameworks to implement them. Since our main goal is to provide a practical method amenable to numerical implementation we highlighted in detail the advantages and shortcomings of the different approaches. We recognized the main ingredients that are required for a practical numerical implementation of this program: i) a set of local dynamical update equations both in space and time, ii) a self-consistent solution of the initial value problem that provides the Cauchy data at the initial time, iii) renormalizability implemented at the level of the update equations, so as to ensure insensitivity to the lattice introduced in the numerical implementation. After analyzing several different manners to implement a variational, self-consistent treatment of the non-equilibrium dynamics for these inhomogeneous problems, we identified a formulation that leads to a closed set of local update equations. We then studied in detail the renormalizability of this set of equations in both one and three spatial dimensions. The study of the dynamics of non-perturbative inhomogeneous field configurations in one spatial dimension is warranted by the interest in understanding the dynamics of soliton configurations in the presence of quantum excitations, but also as model systems to test these methods in simpler settings. In one spatial dimension we provide simple self-consistent ansatz for the initial Cauchy data in terms of exactly solvable potentials for the Sine-Gordon and $\phi^{4}$ model. In the Sine Gordon case the exact mass spectrum, S-matrix, form factors are known and this allowed us to compare this simple ansatz to exact quantum results. Numerical resolution of the full set of variational equations should provide better approximations.

In three dimensions we focused on the linear sigma model in the large $N$ limit as a model for relaxation of non-perturbative coherent pion configurations such as disoriented chiral condensates. In this case we provided a self-consistent solution for the initial Cauchy data in the case of cylindrical symmetry by extending the results from the one dimensional examples.

We found that for large amplitude configurations a mechanism akin to the Klein paradox resulting in particle production is responsible for novel spinodal instabilities that will lead to profuse pion production at low $p_{t}$ through the relaxation of the inhomogeneous condensate.

Furthermore we provided the full set of renormalized (semi)local update equations that can be implemented numerically and discussed in detail the strategy for a practical implementation, which is the next step of the program and currently in progress.

\section{ACKNOWLEDGMENTS}

D. B. thanks the N.S.F for partial support through the grant awards: PHY-9605186 and LPTHE for warm hospitality. F. C thanks the DOE for support, P. S. thanks the INFN for support. F.C. would like to thank Emil Mottola and Salman Habib for several useful discussions and A. Kerman for valuable conversations.

D. B. and H. J. de V. acknowledge partial support from NATO. 


\section{REFERENCES}

[1] V. A. Kuzmin, V. A. Rubakov, and M. E. Shaposhnikov, Phys. Lett. B155, 36 (1985), for reviews on the subject see: A. D. Dolgov, Phys. Repts. 222, 309 (1992); A. G. Cohen, D. B. Kaplan and A. E. Nelson, Annu. REv. Nucl. Part. Sci. 43, 27 (1993).

[2] C. Rebbi and R. Singleton, Phys.Rev. D54 (1996) 1020.

[3] For a review see: H. Meyer-Ortmanns, Rev. of Mod. Phys. 68, 473, (1996).

[4] B. Muller, The Physics of the Quark Gluon Plasma Lecture Notes in Physics, Vol. 225 (Springer-Verlag, Berlin, Heidelberg, 1985).

[5] M. B. Hindmarsh and T.W.B. Kibble, Rep. Prog. Phys. 58:477 (1995).

[6] A. Vilenkin and E.P.S. Shellard, 'Cosmic Strings and other Topological Defects', Cambridge Monographs on Math. Phys. (Cambridge Univ. Press, 1994).

[7] A. Anselm, Phys. Letters B217, 169 (1989); A. Anselm and M. Ryskin,Phys. Letters B226, 482 (1991).

[8] J. P. Blaizot and A. Krzywicki, Phys. Rev. D46, 246 (1992); Acta Phys.Polon.27, 16871702 (1996) and references therein.

[9] J. D. Bjorken, Int. Jour. of Mod. Phys. A7, 4189 (1992); Acta Physica Polonica B23, 561 (1992). See also J. D. Bjorken's contribution to the proceedings of the ECT workshop on Disoriented Chiral Condensates, available at http://www.cern.ch/WA98/DCQ; G. Amelino Camelia, J. D. Bjorken and S. E. Larsson,Phys.Rev. D56 (1997) 6942.

[10] K. L. Kowalski and C. C. Taylor, "Disoriented Chiral Condensate: A white paper for the Full Acceptance Detector, CWRUTH-92-6; J. D. Bjorken, K. L. Kowalski and C. C. Taylor: 'Observing Disoriented Chiral Condensates', (SLAC-CASE WESTERN preprint 1993) hep-ph/9309235; 'Baked Alaska', (SLAC-PUB-6109) (1993).

[11] C.R. Hu, S. Matinyan, B. Muller, Phys.Rev. D54 (1996) 2175, C. R. Hu, S. G. Matinyan, B. Muller, D. Sweet,Phys. Rev. D53 (1996) 3823, C. Hu, S. Matinyan, B. Muller, A. Trayanov, T. Gould, S. Hsu, E. Poppitz, Phys. Rev. D52 (1995) 2402.

[12] A. J. Heeger, S. Kivelson, J. R. Schrieffer and W. P. Su, Rev. Mod. Phys. 60, 781, (1988).

[13] Yu Lu, 'Solitons and Polarons in Conducting Polymers' (World Scientific, Singapore, 1988).

[14] G. Gruner, 'Density Waves in Solids', Addison Wesley (1994), Rev. Mod. Phys. 60, 1129 (1988).

[15] E.J. Copeland, M. Gleiser, H.-R. Mueller, Phys. Rev. D52 (1995) 1920.

[16] K. Rajagopal and F. Wilczek, Nucl. Phys. B404, 577 (1993); K. Rajagopal in "Quark Gluon Plasma 2", R. Hwa, ed. (World Scientific)(1995); K. Rajagopal, hep-ph/9504310; K. Rajagopal, hep-ph/9703258.

[17] S. Gavin, A. Goksch and R. D. Pisarski, Phys. Rev. Lett., 72,2143 (1994); S. Gavin and B. Muller, Phys. Lett. B329, 486 (1994).

[18] M. Asakawa, Z. Huang and X.-N. Wang, Phys. Rev. Lett. 74, 3126 (1995).

[19] J. Randrup, Phys. Rev. Lett. 77, 1226 (1996), Nucl.Phys. A616 (1997) 531, Phys.Rev. D55 (1997) 1188, hep-ph/961122 (unpublished).

[20] D. Vautherin and T. Matsui, Phys. Rev. D 55, 4492, (1997).

[21] D. Boyanovsky and H.J. de Vega, Phys. Rev. D47, 2343 (1993)

[22] D. Boyanovsky, M. D'Attanasio, H.J. de Vega, R. Holman Phys.Rev. D54 (1996) 1748.

[23] C. Bender, F. Cooper and G. Guralnik, Ann. Phys. (N.Y.) 109, 165 (1977); F. Cooper, 
S.-Y. Pi and P. Stancioff, Phys. Rev. D, 34, 3831 (1986); F. Cooper and S. -Y. Pi in Current Trends in Physics Ed. by A. Khare and T. Pradhan (World Scientific, Singapore, 1986).

[24] F. Cooper and E. Mottola, Phys. Rev. D36, 3114 (1987); S.Y. Pi and M. Samiullah, Phys. Ref. D 36, 3128 (1987)

[25] F. Cooper, S. Habib, Y. Kluger, E. Mottola, J.P. Paz, and P.R. Anderson, Phys. Rev. D50, 2848 (1994)

[26] S. Habib, Y. Kluger, E. Mottola, and J.P. Paz, Phys. Rev. Lett. 76 (1996) 4660.

[27] F. Cooper, S. Habib, Y. Kluger, and E. Mottola, Phys.Rev. D55 (1997) 6471.

[28] D. Boyanovsky, D.-S. Lee and A. Singh, Phys. Rev. D48, 800, (1993).

[29] D. Boyanovsky, H.J. de Vega, R. Holman, Phys. Rev. D49, 2769, (1994).

[30] D. Boyanovsky, H. J. de Vega, R. Holman, D S Lee and A Singh,

Phys. Rev. D51, 4419 (1995).

D. Boyanovsky, H. J. de Vega, R. Holman, J. F. J. Salgado,

Phys. Rev. D54, 7570 (1996);

D. Boyanovsky, D. Cormier, H. J. de Vega, R. Holman, Phys. Rev. D55, 3373 (1997).

[31] D. Boyanovsky, H. J. de Vega and R. Holman, 'Erice Lectures on Inflationary Cosmology', p. 183-270 in the Proceedings of the 5th Erice Chalonge School on Astrofundamental Physics, Ed. N. Sanchez, World Scientific, Singapore, 1997, hep-ph-9701304.

[32] D. Boyanovsky, C. Destri, H.J. de Vega, R. Holman, J. F. J. Salgado, 'Asymptotic Dynamics in Scalar Field Theory: Anomalous Relaxation', hep-ph/9711384.

[33] F. Cooper, Y. Kluger, E. Mottola and J. P. Paz, Phys. Rev. D51, 2377 (1995); Y. Kluger, F. Cooper, E. Mottola, J. P. Paz and A. Kovner, Nucl. Phys. A590, 581c (1995); M. A. Lampert, J. F. Dawson and F. Cooper, Phys. Rev. D54, 2213-2221 (1996), F. Cooper, Y. Kluger and E. Mottola, Phys. Rev. C 54, 3298 (1996).

[34] D. Boyanovsky, H.J. de Vega and R. Holman, Phys. Rev. D51, 734 (1995).

[35] Daniel Boyanovsky, Hector J. de Vega, Richard Holman, S. Prem Kumar, Robert D. Pisarski, 'Non-equilibrium evolution of a 'Tsunami': Dynamical Symmetry Breaking ', hep-ph/9711258, to be published in Phys. Rev. D.

[36] P. Dirac. Proc. Camb. Phil. Soc. 26, 376 (1930); A.K. Kerman and S.E. Koonin, Ann. Phys. 100, 332 (1976); R. Jackiw and A. K. Kerman, Phys. Lett. A71, 158 (1979); J. Cornwall, R. Jackiw and E. Tomboulis, Phys. Rev. D10, 2428 (1974); O. Eboli, R. Jackiw and S. Y. Pi, Phys. Rev. D37, 3557 (1988).

[37] P. M. Stevenson, Phys. Rev. D32, 1389, (1985).

[38] A. K. Kerman, C.-Y. Lin, Ann. of Phys. (N.Y.) 241185 (1995); A. K. Kerman, C. Martin and D. Vautherin, Phys. Rev. D47, 632 (1993).

[39] G. Amelino-Camelia, Phys. Rev. D49 (1994) 2740; G. Amelino-Camelia and S.-Y. Pi, Phys. Rev. D47 (1993) 2356.

[40] F. Cooper, G. Frye and E. Schonberg, Phys. Rev. D11, 192, (1975); F. Cooper and D. H. Sharp, Phys. Rev. D12, 1123, (1975); F. Cooper and M. Feigenbaum, Phys. Rev. D14, 583, (1976).

[41] J. D. Bjorken, Phys. Rev. D 27, 140 (1983).

[42] L. P. Csernai, 'Introduction to Relativistic Heavy Ion Collisions', (John Wiley and Sons, England, 1994); C. Y. Wong, 'Introduction to High-Energy Heavy Ion Collisions', (World Scientific, Singapore, 1994). 
[43] B. Mihaila, J. F. Dawson, F.Cooper, Phys. Rev.D 56,5400 (1997). hep-ph/9705354

[44] J. Schwinger, J. Math. Phys. 2, 407 (1961); K. T. Mahanthappa, Phys. Rev. 126, 329 (1962); P. M. Bakshi and K. T. Mahanthappa, J. Math. Phys. 41, 12 (1963); L. V. Keldysh, JETP 20, 1018 (1965); K. Chou, Z. Su, B. Hao And L. Yu, Phys. Rep. 118, 1 (1985); A. Niemi and G. Semenoff, Ann. of Phys. (NY) 152, 105 (1984); N. P. Landsmann and C. G. van Weert, Phys. Rep. 145, 141 (1987); E. Calzetta and B. L. Hu, Phys. Rev. D41, 495 (1990); ibid D37, 2838 (1990); J. P. Paz, Phys. Rev. D41, 1054 (1990); ibid D42, 529(1990); E. Calzetta and B. L. Hu, Phys. Rev. D35, 495 (1987); Phys. Rev. D40, 656 (1989) and references therein.

[45] F. Cooper and E. Mottola, Mod. Phys. Lett. A2,635 (1987)

[46] J. Baacke and H. J. Rothe, Nucl. Phys. B125, 108 (1977); Nucl. Phys. B118, 371 (1977).

[47] J. Rubinstein, J. Math. Phys. 11, 258 (1970)

[48] L.D. Faddev and V.E. Korepin, Phys. Lett. 63B, 435, (1976) and Phys. Rept. 42, 1, (1978).

[49] S. Coleman, Phys. Rev. D11 2088 (1975).

[50] R. Rajaraman, 'Solitons and Instantons'.

[51] A. B. and Al. B. Zamolodchikov, Ann. Phys. 120, 253, (1979).

H. B. Thacker, Rev. Mod. Phys. 53, 253, (1981).

C. Destri and H J de Vega, Nucl. Phys. B 290, 363 (1987).

[52] D. J. Amit, Y. Y. Goldschmidt and G. Grinstein, J. Phys. A13, 585, (1980).

[53] P. Morse and H. Feshbach, 'Methods of Mathematical Physics', (McGraw-Hill), p.1650 (1953).

[54] P. H. Weisz, Phys. Lett. B67, 179 (1977). M. Karowski and P. H. Weisz, Nucl. Phys. B139, 455 (1978).

[55] F. A. Smirnov, Form Factors in Completely Integrable models in QFT, Adv. Series in Mathematics, vol. 14, World Scientific, 1992.

[56] S. J. Chang, Phys. Rev. D 12, 1071 (1975); Phys. Rev. D 13, 2778 (1976)

[57] T.D. Lee and Y. Pang Phys. Rept. 221, 251-350,(1992)

[58] J. L. Gervais and B. Sakita, Phys. Rev. D11 2943 (1975).

[59] J. L. Gervais, A. Jevicki and B. Sakita, Phys. Rev. D12 1038 (1975).

[60] N. H. Christ and T. D. Lee, Phys. Rev. D12 1606 (1975).

[61] E. Tomboulis, Phys. Rev. D12 1678 (1975).

[62] J. L. Gervais and A. Jevicki, Nucl. Phys.B 93, 113 (1976).

[63] H. J. de Vega, Nucl. Phys. B 115, 411 (1976).

[64] S. M. Alamoudi, D. Boyanovsky and F. I. Takakura, cond-mat/9708225 (to appear in Phys. Rev. B 1998) and hep-ph/9710359.

[65] J. Baacke, K. Heitmann, C. Patzold, Phys. Rev. D56 (1997) 6556; Phys. Rev. D55 (1997) 2320; Phys. Rev. D55 (1997) 7815; hep-th/9711144. 


\section{FIGURES}

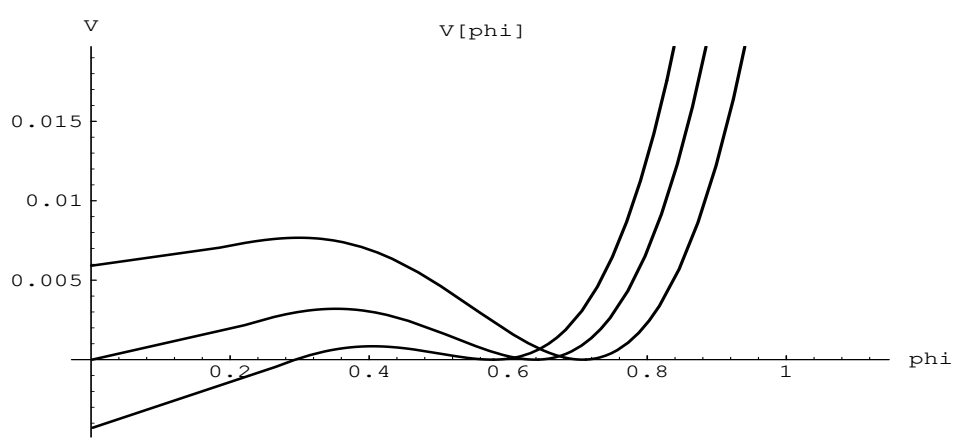

FIG. 1. Variational effective potential for $\phi^{4}$ in $1+1$ dimensions for $g / m_{r}^{2}=1, g_{c}, 2$ 


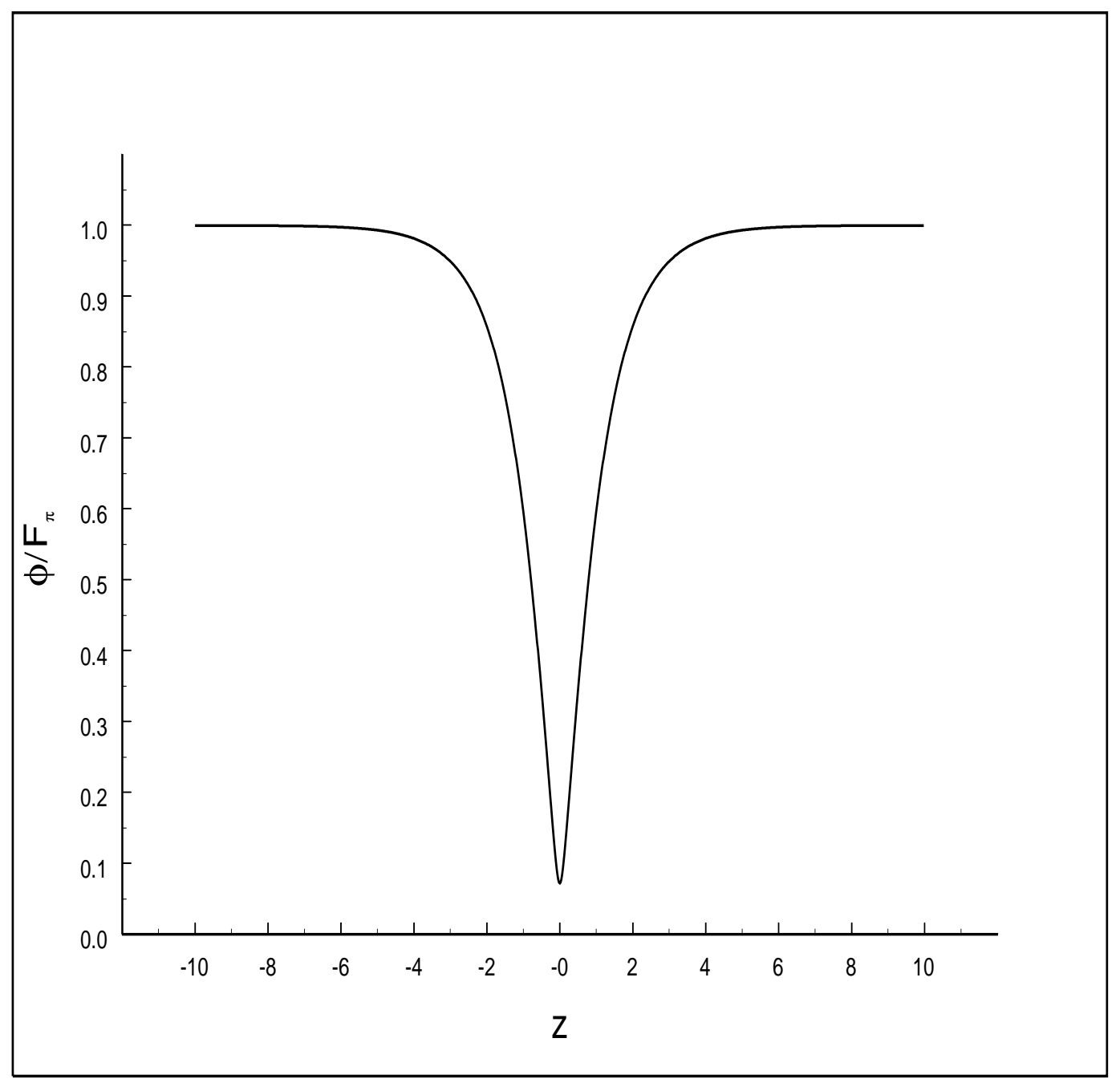

FIG. 2. $\varphi_{c}(z) / F_{\pi}$ vs. $m z$ for $M_{\pi} / m=0.575, \mu=0 \lambda_{R}=36$ 


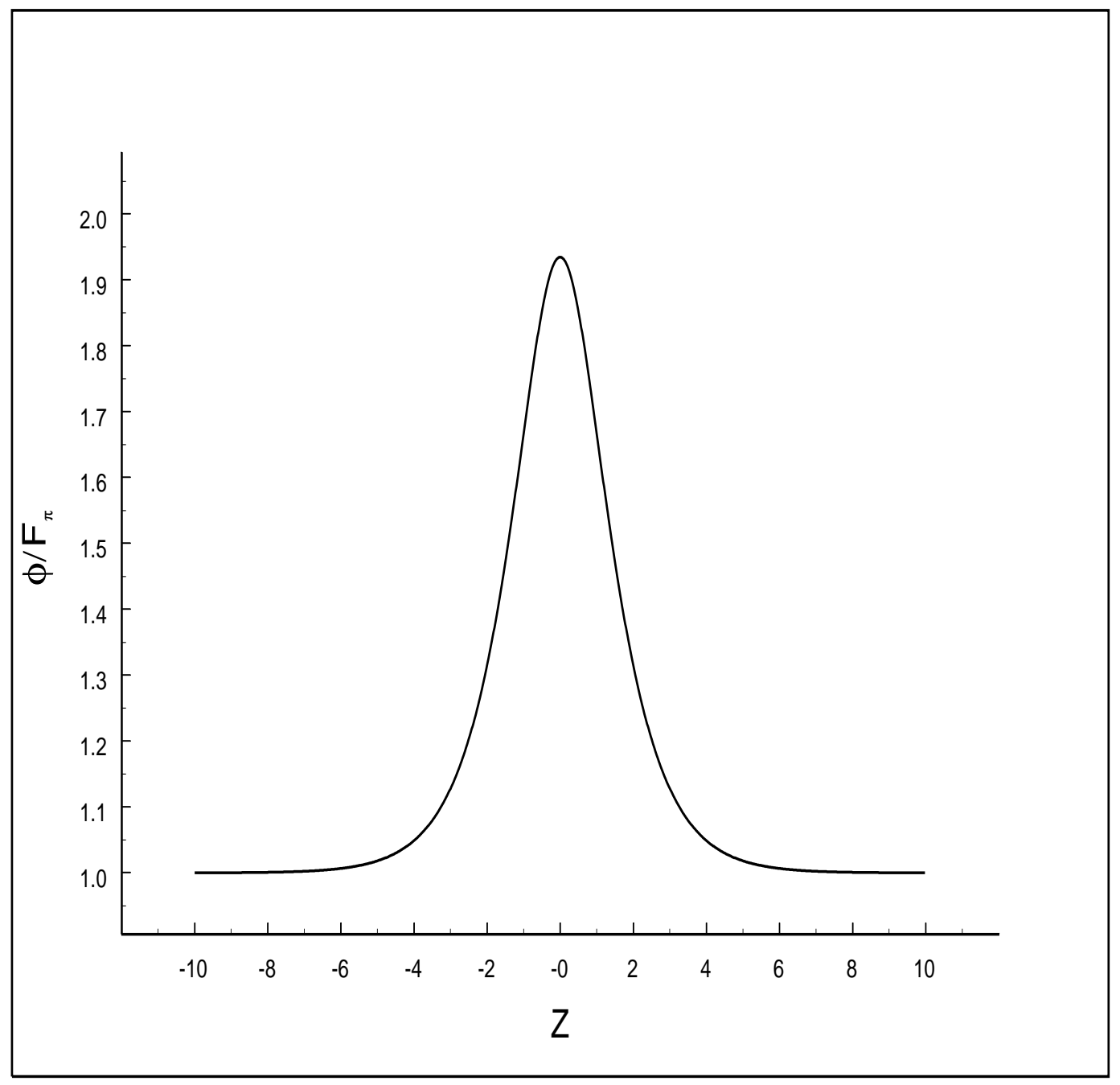

FIG. 3. $\varphi_{c}(z) / F_{\pi}$ vs. $m z$ for $M_{\pi} / m=0.0019, \mu=0, \lambda_{R}=36$ 